\title{
OBSERVATIONALLY-INDUCED ALGEBRAS IN DOMAIN THEORY
}

\author{
INGO BATTENFELD $^{a}$, KLAUS KEIMEL $^{b}$, AND THOMAS STREICHER $^{c}$ \\ ${ }^{a}$ Grotenbachstr. 8, Dortmund, D-44225, Germany \\ e-mail address: ingo-battenfeld@email.de \\ ${ }^{b, c}$ Fachbereich 4 Mathematik TU Darmstadt, Schloßgartenstr. 7, D-64289, Germany \\ e-mail address: \{keimel,streicher\}@mathematik.tu-darmstadt.de
}

\begin{abstract}
In this paper we revise and simplify the notion of observationally induced algebra introduced by Simpson and Schröder for the purpose of modelling computational effects in the particular case where the ambient category is given by classical domain theory.

As examples of the general framework we consider the various powerdomains. For the particular case of the Plotkin powerdomain the general recipe leads to a somewhat unexpected result which, however, makes sense from a Computer Science perspective. We analyze this "deviation" and show how to reobtain the original Plotkin powerdomain by imposing further conditions previously considered by R. Heckmann and J. GoubaultLarrecq.
\end{abstract}

\section{INTRODUCTION}

E. Moggi in his seminal paper 19 described how to model computational effects via so-called "computational monads". Later Power and Plotkin suggested to model computational effects as free algebras which after all give rise to monads. Though some effects like continuations cannot be modeled this way their approach covers most examples of computational effects as described in [20]. In their account the algebras of interest are specified by equational and inequational laws. As an alternative A. Simpson and M. Schröder in [22] suggested to specify classes of algebras not in an axiomatic way but instead by exhibiting a prototypical such algebra $\underline{R}$. For such an algebra $\underline{R}$ one may define a notion of $\underline{R}$-complete algebra. In [22, 2, 3, 4, it is shown how to construct for every object $X$ of the ambient category (of domains) a free $\underline{R}$-complete algebra $\underline{R}(X)$ over $X$, called the repletion of $X$.

The notion of $\underline{R}$-complete algebra is defined in analogy with M. Hyland's notion of replete object as introduced in [11] for the purpose of providing an appropriate notion of completeness within Synthetic Domain Theory. Actually, in case of an empty signature $\underline{R}$-complete algebras coincide with $R$-replete objects in the sense of [11]. The definition

2012 ACM CCS: [Theory of computation]: Logic; Semantics and reasoning-Program semanticsDenotational semantics.

Key words and phrases: denotational semantics, computational effects, powerdomains, domain theory.

Work supported by Deutsche Forschungsgemeinschaft (DFG).

넫임
DOI:10.2168/LMCS-10(3:18)2014

(C) I. Battenfeld, K. Keimel, and T. Streicher Creative Commons 
of $\underline{R}$-completeness in [22, 2, 3, 4] appears as somewhat convoluted because categories of algebras are typically not cartesian closed and, moreover, in some of the cases considered in loc.cit. the ambient category was not cartesian closed as e.g. the category of topological spaces and continuous maps. We assume our ambient category to be cartesian closed and the category of algebras to be enriched over this ambient category. This allows us to come up in section 1 with a notion of completeness which is closer in spirit to Hyland's original notion of repleteness. Moreover, as shown in section 2 the complete algebras form a full reflective subcategory when the ambient category are directed complete partial orders and Scott continuous maps as studied e.g. in [1].

In the remaining sections we study particular cases of computational effects corresponding to the various notions of powerdomains. If the computational effect is given by the computational monad $T$ then the corresponding prototypical algebra $\underline{R}$ is chosen to be $T(\Sigma)$ where $\Sigma$ is the Sierpiński domain. In most cases $\underline{R}(X)$ turns out to coincide with the respective powerdomain of $X$. A notable exception is the Plotkin powerdomain $\mathcal{P}$ in which case $\mathbb{A}=\mathcal{P}(\Sigma)$ is the 3-element chain $\perp \sqsubset m \sqsubset \top$ whose elements stand for "must diverge", "may diverge or converge" and "must converge", respectively. It turns out that for (reasonable) domains $X$ their repletion $\mathbb{A}(X)$ consists of "formal lenses" $(C, Q)$ where

$C$ is Scott closed in $X, Q$ is compact saturated in $X$ and $C \cap Q$ is nonempty. Obviously, in general such formal lenses $(C, Q)$ are not determined by the "real lens" $C \cap Q$. We also give characterizations of the "real" lenses as particular "formal" lenses using and adapting ideas from [10, 8, 9]. However, formal lenses $(C, Q)$ appear as quite natural from a Computer Science perspective since $C$ may be understood as a "safety" predicate and $Q$ as a "liveness" predicate on $X$.

\section{Complete algebras in CARtesian Closed Categories}

We assume $\mathcal{C}$ to be a cartesian closed category and $\Omega$ a finitary algebraic signature, that is, a collection of operation symbols $\omega$ each coming with a finite arity $n=n_{\omega} \in \mathbb{N}$. A $\mathcal{C}_{\Omega}$-algebra in $\mathcal{C}$ is an object $A$ of $\mathcal{C}$ together with $\mathcal{C}$-morphisms $\omega_{A}: A^{n_{\omega}} \rightarrow A$, one for each operation symbol $\omega \in \Omega$. We denote $\mathcal{C}_{\Omega}$-algebras by $\underline{A}, \underline{B}, \ldots$ and by $A, B, \ldots$ the underlying $\mathcal{C}$-objects. A $\mathcal{C}_{\Omega}$-homomorphism between $\mathcal{C}_{\Omega}$-algebras $\underline{A}$ and $\underline{B}$ is a $\mathcal{C}$-morphism $h: A \rightarrow B$ such that $h \circ \omega_{A}=\omega_{B} \circ h^{n}$ for every $\omega \in \Omega$ of arity $n$. We denote by $\mathcal{C}_{\Omega}$ the category of $\mathcal{C}_{\Omega}$-algebras and $\mathcal{C}_{\Omega}$-homomorphisms.

As $\mathcal{C}$ is cartesian closed, the category $\mathcal{C}_{\Omega}$ has $\mathcal{C}$-powers, i.e. for $\underline{A}$ in $\mathcal{C}_{\Omega}$ and $X$ in $\mathcal{C}$ the power $\underline{A}^{X}$ is the algebra whose underlying object is $A^{X}$ and whose operations are defined pointwise as $\omega_{A^{X}}(\vec{u})=\lambda x: X \cdot \omega_{A}(\vec{u}(x))$.

Moreover, we assume $\mathcal{C}_{\Omega}$ to be $\mathcal{C}$-enriched in the sense that for $\mathcal{C}_{\Omega}$-algebras $\underline{A}$ and $\underline{B}$ there is an object $\operatorname{hom}(\underline{A}, \underline{B})$ in $\mathcal{C}$ with $\mathcal{C}_{\Omega}\left(\underline{A}, \mathbb{B}^{X}\right) \cong \mathcal{C}(X, \operatorname{hom}(\underline{A}, \underline{B})$ naturally in $X$. If $\mathcal{C}$ has enough limits hom $(\underline{A}, \underline{B})$ arises as subobject of the exponential $B^{A}$ via an appropriate equalizer.

Finally, we fix a $\mathcal{C}_{\Omega}$-algebra $\underline{R}$ as computational prototype. As originally suggested by A. Simpson and M. Schröder [22] we will define notions of $\underline{R}$-complete algebras which in case of empty signature coincide with the notion of an $R$-replete object [11]. We present two such notions which, however, give rise to the same construction when reflecting free algebras to the $\underline{R}$-complete ones.

First we give the definitions originally suggested in [22, 2, 3, 4] but formulated in a way making use of the assumption that $\mathcal{C}_{\Omega}$ is enriched over $\mathcal{C}$. 
Definition 1.1. Let $X$ be an object of $\mathcal{C}$ and $\underline{A}$ and $\underline{C}$ be algebras in C. A morphism $e: X \rightarrow A$ is called $\underline{C}$-equable, if the restriction of the $\mathcal{C}$-morphism $C^{e}: C^{A} \rightarrow C^{X}$ to $\operatorname{hom}(\underline{A}, \underline{C})$, i.e. $C^{e}: \operatorname{hom}(\underline{A}, \underline{C}) \rightarrow C^{X}: h \mapsto h \circ e$ is an isomorphism in $\mathcal{C}$.

Next, we identify our class of computation types as $\underline{R}$-complete algebras in the following sense.

Definition 1.2. A weakly $\underline{R}$-complete algebra is a $\mathcal{C}_{\Omega}$-algebra $\underline{C}$ such that every $\underline{R}$-equable morphism is also $\underline{C}$-equable. We denote the category of $\mathcal{C}_{\Omega}$-homomorphisms between weakly $\underline{R}$-complete algebras by $w \mathcal{C}_{\underline{R}}$.

Next we give the second stronger version of $\underline{R}$-completeness which has been proposed recently by Battenfeld in [3] (following a suggestion on p.64 of [24]) and is closer in spirit to Hyland's original definition of $R$-replete objects, see [11].

Definition 1.3. For $\mathcal{C}_{\Omega}$-algebras $\underline{A}, \underline{B}$ and $\underline{C}$, a $\mathcal{C}_{\Omega}$-homomorphism $e: \underline{A} \rightarrow \underline{B}$ is called $\underline{C}$-equable, if the $\mathcal{C}$-map $\operatorname{hom}(e, \underline{C}): \operatorname{hom}(\underline{B}, \underline{C}) \rightarrow \operatorname{hom}(\underline{A}, \underline{C}): h \mapsto h \circ e$ is an isomorphism in $\mathcal{C}$.

Definition 1.4. A $\mathcal{C}_{\Omega}$-algebra $\underline{C}$ is called $\underline{R}$-complete if every $\underline{R}$-equable homomorphism $e: \underline{A} \rightarrow \underline{B}$ is also $\underline{C}$-equable. We denote the category of $\underline{R}$-complete $\mathcal{C}_{\Omega^{-}}$-algebras and $\mathcal{C}_{\Omega^{-}}$ homomorphisms by $\mathcal{C}_{\underline{R}}$.

We now discuss why $\underline{R}$-completeness is presumably stronger than weak $\underline{R}$-completeness and why the difference doesn't matter too much for our purposes. For this, we suppose that for every object $X$ in the category $\mathcal{C}$ there is an (absolutely) free $\Omega$-algebra over $X$ in $\mathcal{C}$, i.e. a $\mathcal{C}_{\Omega}$-algebra $\mathcal{F}_{\Omega}(X)$ together with a $\mathcal{C}$-map $i_{X}: X \rightarrow \mathcal{F}_{\Omega}(X)$ such that for every algebra $\underline{A}$ the map $\underline{A}^{i_{X}}: \operatorname{hom}\left(\mathcal{F}_{\Omega}(X), \underline{A}\right) \rightarrow A^{X}: h \mapsto h \circ i_{X}$ is an isomorphism in $\mathcal{C}$. This amounts to an internalization to $\mathcal{C}$ of the requirement that for every morphism $e: X \rightarrow A$ there is a unique homomorphic extension $\widehat{e}: \mathcal{F}_{\Omega}(X) \rightarrow \underline{A}$ along $i_{X}$ as depicted in

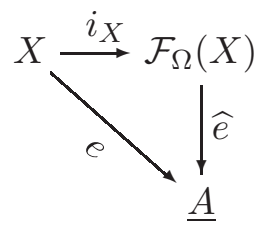

Thus, a morphism $e: X \rightarrow A$ is $\underline{C}$-equable in the sense of Def. 1.1 iff the homomorphic extension $\widehat{e}: \mathcal{F}_{\Omega}(X) \rightarrow \underline{A}$ is $\underline{C}$-equable in the sense of Definition 1.3. For this reason, an $\underline{R}$-complete algebra according to Definition 1.4 is in particular also weakly $\underline{R}$-complete in the sense of Definition 1.2 .

The notion of completeness has the advantage that for interesting instances of $\mathcal{C}$, as e.g. the category $\mathcal{D}$ cpo of directed complete posets, the $\underline{R}$-complete algebras form a full reflective subcategory of $\mathcal{C}_{\Omega}$. But, as we will show later, in the category $\mathcal{D}$ cpo for every object $X$ the free weakly $\underline{R}$-complete algebra over $X$ coincides with the reflection of $\mathcal{F}_{\Omega}(X)$ to the category $\mathcal{C}_{\underline{R}}$ of $\underline{R}$-complete algebras.

We recall for later use that the forgetful functor $\mathcal{C}_{\Omega} \rightarrow \mathcal{C}$ creates limits. It turns out that the same holds for both of the subcategories of weakly $\underline{R}$-complete and also $\underline{R}$-complete algebras.

Lemma 1.5. The forgetful functor $U: w \mathcal{C}_{\underline{R}} \rightarrow \mathcal{C}$ creates limits. The same holds for the category of $\underline{R}$-complete algebras. 
Proof. Clearly, the forgetful functor factors as $\mathcal{C}_{\underline{R}} \rightarrow \mathcal{C}_{\Omega} \rightarrow \mathcal{C}$. The forgetful functor $\mathcal{C}_{\Omega} \rightarrow \mathcal{C}$ is known to create limits. Thus, if $D$ is a diagram in $\mathcal{C}_{\underline{R}}$, and the limit of $U \circ D$ exists in $\mathcal{C}$, then its $\operatorname{limit} \operatorname{Lim}(D)$ carries a canonical $\Omega$-algebra structure, making $\left(\operatorname{Lim}(D),\left\{\omega_{\operatorname{Lim}(D)}\right\}\right)$ the corresponding limit in $\mathcal{C}_{\Omega}$. It only remains to show that $\left(\operatorname{Lim}(D),\left\{\omega_{\operatorname{Lim}(D)}\right\}\right)$ is (weakly) $\underline{R}$-complete, which follows from a straightforward calculation.

\section{Complete algebras in Classical Domain Theory}

We now study the notions of the previous section for the classical case where $\mathcal{C}$ is the category Dcpo of directed complete partial orders and Scott continuous maps between them. We start by fixing our notation.

Recall that a partially ordered set is directed complete if every directed family $\left(x_{i}\right)_{i \in I}$ of elements has a supremum that we denote by $\bigvee_{i \in I}^{\uparrow} x_{i}$ in order to indicate that it is the supremum of a directed family. A map $f$ between dcpos is continuous (in the sense of Scott) if it preserves the order and suprema of directed families. It is well-known that the category Dcpo is cartesian closed, complete and cocomplete (see e.g. [1]). The exponential, denoted by $Y^{X}$ and alternatively by $[X \rightarrow Y]$, is given by the set of Scott-continuous functions $u: X \rightarrow Y$ ordered pointwise. Suprema of directed families in $Y^{X}$ are computed pointwise. A subset $Y$ of a dcpo $X$ is said to be a sub-dcpo if, for every directed family $\left(y_{i}\right)_{i \in I}$ of elements in $Y$, the supremum $\bigvee_{i \in I}^{\uparrow} y_{i}$ (taken in $X$ ) belongs to $Y$.

As in the previous section, we fix a finitary algebraic signature $\Omega$. A $\mathcal{D}_{c p o} \Omega_{\Omega^{-a l g e b r a ~}} \underline{A}$ is a dcpo $A$ together with continuous operations $\omega_{A}: A^{n} \rightarrow A$ for every $\omega \in \Omega$ with arity $n$. A map $h: \underline{A} \rightarrow \underline{B}$ between $\mathcal{D} c o_{\Omega}$-algebras is an $\Omega$-homomorphism if

$$
h\left(\omega_{A}\left(a_{1}, \ldots, a_{n}\right)\right)=\omega_{B}\left(h\left(a_{1}\right), \ldots, h\left(a_{n}\right)\right)
$$

for every $\omega \in \Omega$ of arity $n$ and all $a_{1}, \ldots, a_{n} \in A$. We write $\mathcal{D} c p o_{\Omega}$ for the category of $\mathcal{D c p o}_{\Omega^{-}}$-algebras and continuous $\Omega$-homomorphisms.

For a directed family of continuous $\Omega$-homomorphisms $\left\{h_{i}: \underline{A} \rightarrow \underline{B}\right\}_{i \in I}$, their (pointwise) supremum $h(x)=\bigvee_{i \in I}^{\uparrow} h_{i}(x)$ is again a continuous $\Omega$-homomorphism $h: \underline{A} \rightarrow \underline{B}$. Hence, the continuous homomorphisms from $\underline{A}$ to $\underline{B}$ give rise to a sub-dcpo hom $(\underline{A}, \underline{B})$ of the exponential $B^{A}$ in $\mathcal{D}$ cpo for which reason the category $\mathcal{D}_{c p o}$ is $\mathcal{D}$ cpo-enriched.

For a dcpo $X$ and a $\mathcal{D}$ cpo $_{\Omega^{-}}$-algebra $\underline{A}$, the exponential $A^{X}$ in $\mathcal{D}$ cpo can be endowed with the structure of a $\mathcal{D} c p o_{\Omega^{-a l g e b r a}}$ by defining the operations $\omega$ on $A^{X}$ as

$$
\omega_{A^{X}}\left(u_{1}, \ldots, u_{n}\right)=\lambda x: X . \omega_{A}\left(u_{1}(x), \ldots, u_{n}(x)\right)
$$

where $n$ is the arity of $\omega$.

It is well-known (see [1]) that for every dcpo $X$ there is an (absolutely) free $\mathcal{D}_{c p o} \Omega^{-}$ algebra over $X$, i.e. a $\mathcal{D} c c_{\Omega}$-algebra $\mathcal{F}_{\Omega}(X)$ together with a continuous map $i_{X}: X \rightarrow$ $\mathcal{F}_{\Omega}(X)$ such that for every continuous map $f$ from $X$ to a $\mathcal{D} c p o_{\Omega}$-algebra $\underline{A}$ there is a 
unique continuous homomorphic extension $\tilde{f}: \underline{\mathcal{F}}_{\Omega}(X) \rightarrow \underline{A}$ of $f$ along $i_{X}$ as in

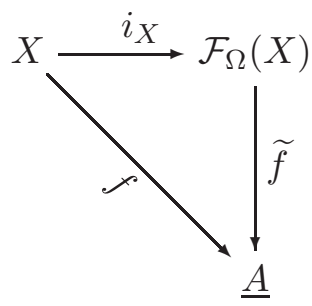

Moreover, the map $f \mapsto \tilde{f}: A^{X} \rightarrow \operatorname{hom}\left(\mathcal{F}_{\Omega}(X), \underline{A}\right)$ is not only bijective but an isomorphism of dcpos (see, e.g. [1]).

We now fix a $\mathcal{D c p o}_{\Omega^{-}}$-algebra $\underline{R}$ as computational prototype and identify our class of computation types as $\underline{R}$-complete algebras as in Section 1 .

Definition 2.1. For $\mathcal{D}^{c p o} o_{\Omega}$-algebras $\underline{A}, \underline{B}$ and $\underline{C}$, a continuous homomorphism $e: \underline{A} \rightarrow \underline{B}$ is called $\underline{C}$-equable, if every continuous homomorphism $h: \underline{A} \rightarrow \underline{C}$ has a unique continuous homomorphic extension $\widehat{h}: \underline{B} \rightarrow \underline{C}$ along $e$ as in

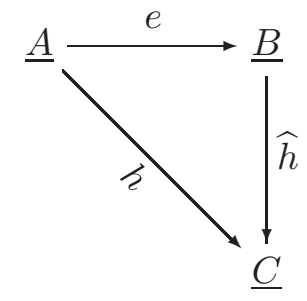

such that the map

$$
h \mapsto \widehat{h}: \operatorname{hom}(\underline{A}, \underline{C}) \rightarrow \operatorname{hom}(\underline{B}, \underline{C})
$$

is an isomorphism.

This definition of "equable" fits under the general scheme of Definition 1.3. First notice that the map $\operatorname{hom}(e, \underline{C}): \operatorname{hom}(\underline{B}, \underline{C}) \rightarrow \operatorname{hom}(\underline{A}, \underline{C})$ is always continuous. It is surjective if and only if every continuous homomorphism $h: \underline{A} \rightarrow \underline{C}$ has at least one continuous homomorphic extension $\widehat{h}: \underline{B} \rightarrow \underline{C}$ along $e$. It is bijective if and only if every continuous homomorphism $h: \underline{A} \rightarrow \underline{C}$ has a unique continuous homomorphic extension $\widehat{h}: \underline{B} \rightarrow \underline{C}$ along $e$. Then the map $\operatorname{hom}(e, \underline{C}): \operatorname{hom}(\underline{B}, \underline{C}) \rightarrow \operatorname{hom}(\underline{A}, \underline{C})$ is an isomorphism within $\mathcal{D} c p o$ iff the inverse map $h \mapsto \widehat{h}$ preserves the order (since isomorphisms in $\mathcal{D}$ cpo are bijective maps which both preserve and reflect the partial order). Thus, the map $e$ is $\underline{C}$-equable if and only if $\operatorname{hom}(e, \underline{C})$ is an isomorphism of dcpos.

Definition 2.2. A $\mathcal{D} c p o_{\Omega^{-}}$-algebra $\underline{C}$ is said to be $\underline{R}$-complete, if every $\underline{R}$-equable $\mathcal{D} c p o_{\Omega^{-}}$ homomorphism $e: \underline{A} \rightarrow \underline{B}$ is also $\underline{C}$-equable. We denote by $\operatorname{Dcpo}_{\underline{R}}$ the category of $\underline{R}-$ complete $\mathcal{D}$ poo $_{\Omega^{-}}$-algebras and $\mathcal{D}$ cpo $_{\Omega}$-homomorphisms.

It is our aim in this section to prove the following theorem.

Theorem 2.3. For every finitary algebraic signature $\Omega$ and every computational prototype $\underline{R}$, the category $\mathcal{D}{ }^{2} o_{\underline{R}}$ of all $\underline{R}$-complete $\mathcal{D}$ cpo $\Omega_{\Omega^{-}}$-algebras is a full reflective subcategory of the category $\mathcal{D}$ cpo $_{\Omega}$ of all $\mathcal{D}$ cpo $_{\Omega}$-algebras.

Recall that for a dcpo $X$ and $\mathcal{D}$ cpo $_{\Omega^{-a l g e b r a s}} \underline{A}$ and $\underline{B}$ the canonical isomorphism $\left(B^{A}\right)^{X} \cong\left(B^{X}\right)^{A}$ restricts to a dcpo-isomorphism hom $(\underline{A}, \underline{B})^{\bar{X}} \cong \operatorname{hom}\left(\underline{A}, \underline{B}^{X}\right)$ which observation will be useful when proving the following lemma. 
Lemma 2.4. A Scott-continuous homomorphism $e: \underline{A} \rightarrow \underline{B}$ is $\underline{C}$-equable if and only if it is $\underline{C}^{X}$-equable for all dcpos $X$.

Proof. Obviously, the backward direction is trivial. For the forward direction suppose that $e: \underline{A} \rightarrow \underline{B}$ is a $\underline{C}$-equable homomorphism and $X$ is an object of $\mathcal{C}$. First notice that the diagram

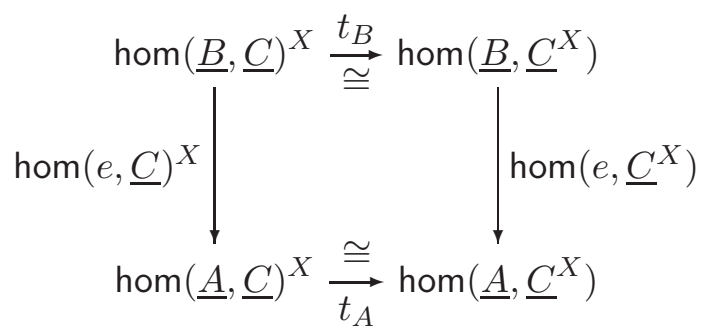

commutes where $t_{A}$ and $t_{B}$ are the canonical isomorphisms which "swap arguments". Since $e$ is $\underline{C}$-equable the left vertical arrow is an isomorphism from which it follows that the right vertical arrow is an isomorphism as well.

From the previous lemma it follows that $\underline{R}$-complete algebras are closed under arbitrary $\mathcal{D}$ cpo-powers. Since $\underline{R}$ clearly is $\underline{R}$-complete, it follows that all $\mathcal{D}$ cpo-powers $\underline{R}^{X}$ are $\underline{R}$ complete.

Corollary 2.5. The category $\mathcal{D}{ }^{2} o_{\underline{R}}$ inherits $\mathcal{D}$ cpo-powers from $\mathcal{D}{ } o_{\Omega}$.

For the next result, let us fix $\mathcal{D} c_{\Omega^{-}}$-algebras $\underline{A}$ and $\underline{B}$. There is a canonical continuous map $\eta: A \rightarrow B^{B^{A}}$, namely the transposition of the identity on $B^{A}$, which in $\lambda$-notation can be written as $\lambda x . \lambda f . f(x)$. Writing $\iota$ for the inclusion of $\operatorname{hom}(\underline{A}, \underline{B})$ into $B^{A}$ we can define the map $B^{\iota} \circ \eta: A \rightarrow B^{\operatorname{hom}(\underline{A}, \underline{B})}$ which we also denote by $\eta$ and in $\lambda$-notation can be written as $\lambda x . \lambda h . h(x)$.

Lemma 2.6. For all $\mathcal{D} c p o_{\Omega}$-algebras $\underline{A}$ and $\underline{B}$, the map $\eta: \underline{A} \rightarrow \underline{B}^{\operatorname{hom}(\underline{A}, \underline{B})}$ is an $\Omega$ homomorphism.

Proof. For $\omega \in \Omega$ with arity $n$ we have

$$
\begin{aligned}
& \eta\left(\omega_{A}\left(a_{1}, \ldots, a_{n}\right)\right)=\left(\lambda x \cdot \lambda h \cdot(h(x))\left(\omega_{A}\left(a_{1}, \ldots, a_{n}\right)\right)\right. \\
& =\lambda h \cdot h\left(\omega_{A}\left(a_{1}, \ldots, a_{n}\right)\right) \\
& =\lambda h \cdot \omega_{A}\left(h\left(a_{1}\right), \ldots, h\left(a_{n}\right)\right) \\
& =\omega_{B \text { hom }(\underline{A}, \underline{B})}\left(\lambda h \cdot h\left(a_{1}\right), \ldots, \lambda h \cdot h\left(a_{n}\right)\right)
\end{aligned}
$$

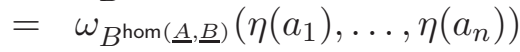

for all $a_{1}, \ldots, a_{n} \in A$.

Thus, in particular, for every $\mathcal{D}_{c p o}{ }_{\Omega^{-}}$algebra $\underline{A}$, we obtain a canonical $\mathcal{D}_{c} o_{\Omega}$-homomor-

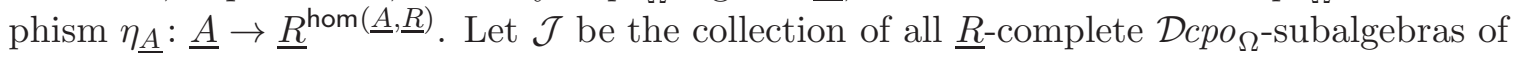
$\underline{R}^{\text {hom }(\underline{A}, \underline{R})}$ containing the image of $A$ under $\eta_{\underline{A}}$. By Corollary 2.5 , the algebra $\underline{R}^{\text {hom }(\underline{A}, \underline{R})}$ is $\underline{R}-$ complete and hence a member of $\mathcal{J}$, so that $\overline{\mathcal{J}}$ is nonempty. We can form the $\overline{\mathcal{D}} c p o_{\Omega}$-algebra $\underline{R}(\underline{A})=\bigcap \mathcal{J}$ and write $r_{\underline{A}}: \underline{A} \rightarrow \underline{R}(\underline{A})$ for the corestriction of $\eta_{\underline{A}}: \underline{A} \rightarrow \underline{R}^{\text {hom }(\underline{A}, \underline{R})}$.

Proposition 2.7. For every $\mathcal{D}$ ppo $_{\Omega}$-algebra $\underline{A}$ we have that

(1) $\underline{R}(\underline{A})$ is $\underline{R}$-complete

(2) $r_{\underline{A}}: \underline{A} \rightarrow \underline{R}(\underline{A})$ is $\underline{R}$-equable. 
Proof. By construction $\underline{R}(\underline{A})$ is a $\mathcal{D} c p o_{\Omega^{-}}$subalgebra of $\underline{R}^{\text {hom }(\underline{A}, \underline{R})}$ containing the image of $\underline{A}$ under $\eta_{A}$. By Lemma 1.5, $\underline{R}(\underline{A})$ is $\underline{R}$-complete, since the intersection of a collection of $\underline{R}$-complete $\mathcal{D} c p o_{\Omega^{-}}$-subalgebras is the limit of the corresponding $\mathcal{D} c p o_{\Omega^{-}}$-subalgebra embeddings.

It remains to show that $r_{\underline{A}}: \underline{A} \rightarrow \underline{R}(\underline{A})$ is $\underline{R}$-equable. For $h \in \operatorname{hom}(\underline{A}, \underline{R})$ let $\ell(h)$ : $\underline{R}(\underline{A}) \rightarrow \underline{R}$ be given by $\lambda f: \underline{R}(\underline{A}) . f(h)$ which is easily seen to be a homomorphism. Moreover, the map $\ell: \operatorname{hom}(\underline{A}, \underline{R}) \rightarrow \operatorname{hom}(\underline{R}(\underline{A}), \underline{R})$ is continuous since it is given by the $\lambda$ term $\lambda h . \lambda f . f(h)$. The homomorphism $\ell(h)$ extends $h$ along $r_{A}$ since $\left(\ell(h) \circ r_{A}\right)(a)=$ $\ell(h)\left(r_{A}(a)\right)=\ell(h)\left(\eta_{A}(a)\right)=\eta_{A}(a)(h)=h(a)$ for all $a \in A$.

We show now that $\ell(h): \underline{R}(\underline{A}) \rightarrow \underline{R}$ is the unique continuous homomorphic extension of $h$ along $r_{\underline{A}}$. For this purpose suppose $g, g^{\prime} \in \operatorname{hom}(\underline{R}(\underline{A}), \underline{R})$ with $g \circ r_{\underline{A}}=h=g^{\prime} \circ r_{\underline{A}}$. Then the equalizer of $g$ and $g^{\prime}$ contains the image of $A$ under $r_{\underline{A}}$ and thus under $\eta_{\underline{A}}$. Since $\underline{R}(\underline{A})$ and $\underline{R}$ are $\underline{R}$-complete, the equalizer is an $\underline{R}$-complete $\mathcal{D}$ cpo $_{\Omega^{-}}$-subalgebra of $\underline{R}(\underline{A})$ by Lemma 1.5 , By construction, $\underline{R}(\underline{A})$ is the smallest $\underline{R}$-complete $\mathcal{D}$ cpo $_{\Omega^{-}}$-subalgebra of $\underline{R}^{\text {hom }(\underline{A}, \underline{R})}$ containing the image of $\eta_{A}$. Thus, the equalizer of $g$ and $g^{\prime}$ must be an isomorphism from which it follows that $g=g^{\prime}$ as desired.

Thus, the map $\ell$ is the continuous inverse of hom $\left(r_{\underline{A}}, \underline{R}\right)$, i.e. $r_{\underline{A}}$ is $\underline{R}$-equable as claimed.

Remark 2.8. More generally, for a homomorphism $e: \underline{A} \rightarrow \underline{B}$ there is a natural correspondence between homomorphisms $h$ making the diagram

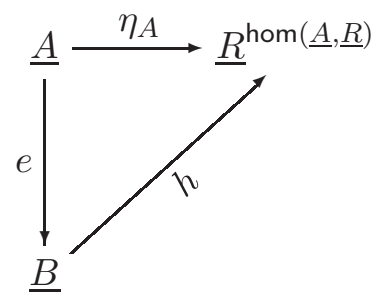

commute and continuous sections $s$ of the $\mathcal{C}$-map hom $(e, \underline{R}): \operatorname{hom}(\underline{B}, \underline{R}) \rightarrow \operatorname{hom}(\underline{A}, \underline{R})$ given by $h(y)(p)=s(p)(y)$ for $y \in B$ and $p \in \operatorname{hom}(\underline{A}, \underline{R})$.

Obviously, such $e$ are $\underline{R}$-equable iff $\operatorname{hom}(e, \underline{R})$ is monic.

Having Proposition 2.7 available we can easily give now the

Proof of Theorem 2.3:

Let $\underline{A}$ be a $\mathcal{D}$ cpo $_{\Omega^{-}}$-algebra. Then $\underline{R}(\underline{A})$ is $\underline{R}$-complete and, since $r_{\underline{A}}: \underline{A} \rightarrow \underline{R}(\underline{A})$ is $\underline{R}$ equable, for every homomorphism $h$ from $\underline{A}$ to an $\underline{R}$-complete algebra $\underline{B}$ there exists a unique homomorphism $\widehat{h}: \underline{R}(\underline{A}) \rightarrow \underline{B}$ with $\widehat{\widehat{h}} \circ r_{\underline{A}}=\underline{h}$.

Definition 2.9. For a $\mathcal{D} c p o_{\Omega^{-a l g e b r a}} \underline{A}$ we call $\underline{R}(\underline{A})$ the $\underline{R}$-repletion of $\underline{A}$ and $r_{\underline{A}}: \underline{A} \rightarrow$ $\underline{R}(\underline{A})$ the reflection map for $\underline{A}$.

One can give the following characterization of $\underline{R}$-repletion which avoids any reference to $\underline{R}$-completeness.

Proposition 2.10. For a $\mathcal{D} c_{\Omega^{-}}$-algebra $\underline{A}$, up to isomorphism $r_{\underline{A}}$ : $\underline{A} \rightarrow \underline{R}(\underline{A})$ is the unique $\underline{R}$-equable homomorphism such that for every $\underline{R}$-equable homomorphism $h: \underline{A} \rightarrow \underline{B}$ there is a unique homomorphism $\widehat{h}: \underline{B} \rightarrow \underline{R}(\underline{A})$ with $\widehat{h} \circ h=r_{\underline{A}}$.

Alternatively, one may characterize $r_{\underline{A}}$ as the up to isomorphism unique $\underline{R}$-equable homomorphism from $\underline{A}$ to an $\underline{R}$-complete algebra. 
Proof. Obviously, the condition of the first characterization determines $r_{\underline{A}}$ uniquely up to isomorphism. That $r_{\underline{A}}$ has the required property is an immediate consequence of the fact that $\underline{R}(\underline{A})$ is $\underline{R}$-complete.

The second characterization follows from the fact that $\underline{R}(\underline{A})$ is $\underline{R}$-complete and that every $\underline{R}$-equable homomorphism $h$ from $\underline{A}$ to an $\underline{R}$-complete algebra satisfies the condition of the first characterization.

According to the alternative Definition 1.1 of $\underline{R}$-equability, for an algebra $\underline{A}$ a map $e: X \rightarrow A$ is $\underline{R}$-equable iff every continuous map $f: X \rightarrow R$ has a unique continuous homomorphic extension $\widehat{f}: \underline{A} \rightarrow \underline{R}$ such that $f \mapsto \widehat{f}: R^{X} \rightarrow \operatorname{hom}(\underline{A}, \underline{R})$ is continuous. Obviously, a map $e: X \rightarrow A$ is $\underline{R}$-equable in the sense of Definition 1.1 if and only if its homomorphic extension $\widehat{e}: \mathcal{F}_{\Omega}(X) \rightarrow \underline{A}$ is $\underline{R}$-equable in the sense of Definition 1.3 ,

For a dcpo $X$ we consider the map $\eta_{X}: X \rightarrow R^{R^{X}}$ defined as $\eta_{X}(x)=\lambda f \cdot f(x)$. Let $w \underline{R}(X)$ be the least weakly $\underline{R}$-complete $\mathcal{D} c p o_{\Omega^{-}}$-subalgebra of $\underline{R}^{R^{X}}$ containing the image of $\eta_{X}$ and $r_{X}^{w}: X \rightarrow w \underline{R}(X)$ be the corestriction of $\eta_{X} \underline{1}$ We call it the weak repletion of $X$. Again one can prove that $r_{X}^{w}$ is $\underline{R}$-equable. Moreover, one easily proves the following analogue of Proposition 2.10.

Proposition 2.11. For a dcpo $X$ up to isomorphism $r_{X}^{w}: X \rightarrow w \underline{R}(X)$ is the unique weakly $\underline{R}$-equable map such that for every weakly $\underline{R}$-equable map $f: X \rightarrow \underline{A}$ there is a unique homomorphism $\widehat{f}: \underline{A} \rightarrow w \underline{R}(X)$ with $\widehat{f} \circ f=r_{X}^{w}$.

Alternatively, one may characterize $r_{X}^{w}$ as the up to isomorphism unique weakly $\underline{R}-$ equable map to a weakly $\underline{R}$-complete algebra.

We do not know whether the weakly $\underline{R}$-complete algebras form a full reflective subcategory of $\mathcal{D} c p o_{\Omega}$. But weak repletion can be understood as repletion of free algebras as we show next.

Proposition 2.12. For every dcpo $X$ the weak repletion $w \underline{R}(X)$ is isomorphic to the repletion $\underline{R}\left(\mathcal{F}_{\Omega}(X)\right)$ of the free $\mathcal{D} c_{\Omega^{-}}$algebra $\mathcal{F}_{\Omega}(X)$ generated by $X$. Moreover, the isomorphism $h: \underline{R}\left(\mathcal{F}_{\Omega}(X)\right) \rightarrow w \underline{R}(X)$ can be chosen in such a way that $h \circ r_{\mathcal{F}_{\Omega}(X)} \circ i_{X}=r_{X}^{w}$.

Proof. Since $\operatorname{hom}\left(\mathcal{F}_{\Omega}(X), \underline{R}\right) \rightarrow R^{X}: h \mapsto h \circ i_{X}$ is an isomorphism in Dcpo the maps $i_{X}$ and $r_{\mathcal{F}_{\Omega}(X)} \circ i_{X}: X \rightarrow w \underline{R}\left(\mathcal{F}_{\Omega}(X)\right)$ are weakly $\underline{R}$-equable. Moreover, since $\underline{R}\left(\mathcal{F}_{\Omega}(X)\right)$ is $\underline{R}$-complete it is in particular weakly $\underline{R}$-complete. Thus, by Proposition 2.11 there is an isomorphism $h: \underline{R}\left(\mathcal{F}_{\Omega}(X)\right) \rightarrow w \underline{R}(X)$ with $h \circ r_{\mathcal{F}_{\Omega}(X)} \circ i_{X}=r_{X}^{w}$.

\section{FREE DCPO-ALGEBRAS}

For semantics, computational effects are mostly modelled by monads (see [19]). For algebraic effects, monads can be specialized to free constructions, an aspect advocated by Plotkin and Power [20]. In the category of dcpos this comes down to consider Dcpo-algebras that are free with respect to a collection of equational and inequational laws that are considered to be characteristic of the computational effect under consideration. This approach is quite different to the observationally-induced approach, to which we want to compare it in this paper.

\footnotetext{
${ }^{1}$ Such a subalgebra exists by an argument analogous to the one in Proposition 2.7
} 
Let $\Omega$ be a finitary signature. If $t_{1}$ and $t_{2}$ are two terms, a $\mathcal{D} c p o_{\Omega^{-a l g e b r a}} \underline{R}$ is said to satisfy the inequational law $t_{1} \leq t_{2}$ (resp., the equational law $t_{1}=t_{2}$ ), if this inequality (resp. equality) holds for every instantiation of the variables by elements of $\underline{R}$. For a given collection $\mathcal{E}$ of equational and inequational laws, denote by $\mathcal{D} c_{\Omega, \mathcal{E}}$ the class of $\mathcal{D}{ } o_{\Omega^{-}}$ algebras that satisfy all the laws in $\mathcal{E}$.

It is well-known that over every dcpo $X$ there is a free $\mathcal{D}{ } o_{\Omega, \mathcal{E}}$-algebra, that is, a

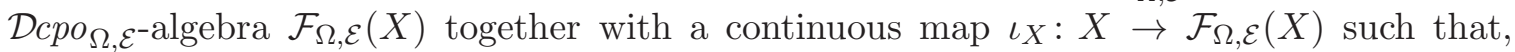
for every continuous map $f$ from $X$ to some $\mathcal{D}^{c p o} o_{\Omega, \mathcal{E}}$-algebra $\underline{A}$, there is a unique $\mathcal{D}{ } o_{\Omega^{-}}$ homomorphism $\tilde{f}: \mathcal{F}_{\Omega, \mathcal{E}}(X) \rightarrow \underline{A}$ such that $f=\tilde{f} \circ \iota_{X}$. This is usually proved using the adjoint functor theorem (see, e.g. [1, Theorem 6.1.2]).

It is desirable for free algebras to be free in the enriched sense as well, namely that $A^{\iota X}: \operatorname{hom}\left(\mathcal{F}_{\Omega, \mathcal{E}}(X), \underline{A}\right) \rightarrow A^{X}$ is an isomorphism of dcpos. For this it is necessary and sufficient that the extension operator $f \mapsto \tilde{f}: A^{X} \rightarrow \operatorname{hom}\left(\mathcal{F}_{\Omega, \mathcal{E}}(X), \underline{A}\right)$ preserves the order. We do not believe that all free dcpo-algebras are free in this enriched sense, although we have no counterexample. But restricting the attention to continuous dcpos and using the description of $\mathcal{F}_{\Omega, \mathcal{E}}(X)$ presented in [1, Section 6.1.2] one can show:

Proposition 3.1. For continuous dcpos $X$, the free $\mathcal{D}_{c p o}{ }_{\Omega, \mathcal{E}}$-algebra $\mathcal{F}_{\Omega, \mathcal{E}}(X)$ always is internally free.

If $\mathcal{F}_{\Omega, \mathcal{E}}(X)$ is internally free, then $\iota_{X}: X \rightarrow \mathcal{F}_{\Omega, \mathcal{E}}(X)$ is $\underline{R}$-equable for every $\mathcal{D}_{c p o}$ s, $^{-}$ algebra $\underline{R}$. Using the characterization of repletion in Proposition 2.11 we obtain the following criterion for the free algebra construction to agree with the observationally induced approach of repletion:

Proposition 3.2. Let $\underline{R}$ be a $\mathcal{D}$ cpo $_{\Omega^{-}}$-algebra satisfying a collection $\mathcal{E}$ of equational and inequational laws. If the free algebra $\iota_{X}: X \rightarrow \mathcal{F}_{\Omega, \mathcal{E}}(X)$ is internally free and $\mathcal{F}_{\Omega, \mathcal{E}}(X)$ is $\underline{R}$-complete, then it is (isomorphic to) the $\underline{R}$-repletion of $X$.

The repletion $r_{X}: X \rightarrow \underline{R}(X)$ of a dcpo $X$ with respect to a computational prototype algebra $\underline{R}$ has some features in common with the free construction $\iota_{X}: X \rightarrow \mathcal{F}_{\Omega, \mathcal{E}}(X)$ with respect to a collection $\mathcal{E}$ of equational and inequational laws. Indeed, the repletion $\underline{R}(X)$ is a subalgebra of a dcpo-power of $\underline{R}$. Since directed suprema in dcpo powers are formed pointwise, $\underline{R}(X)$ satisfies all equational and inequational laws that hold in $\underline{R}$. Thus, a first necessary condition for $\underline{R}(X)$ and $\mathcal{F}_{\Omega, \mathcal{E}}(X)$ to agree is that all equational and inequational laws that hold in $\underline{R}$ can be derived from those in $\mathcal{E}$. But this condition is by no means sufficient. It is not easy to illustrate these phenomena. Already the free algebras are difficult to describe explicitly, while it is even more difficult to put one's hands on the repletion.

We will illustrate these phenomena in the relatively simple situation of the classical powerdomains often named after Hoare, Smyth and Plotkin which model angelic, demonic and erratic nondeterminism, respectively. We will see that the Hoare and Smyth powerdomains agree with the repletion with respect to the natural domains of observation, while there is a big gap between both for the Plotkin powerdomain. There seems to be an intriguing connection to the fact that the inequational theories used in the case of angelic and demonic nondeterminism are complete in the sense that adding any inequational law that is not derivable leads to inconsistency; the inequational theory used in the erratic case has exactly two consistent extensions, namely the inequational theories used for the angelic and demonic cases. But we do not want to develop this observation any further here. 


\section{Examples: Powerdomain Constructions}

We now examine whether the classical powerdomain constructions can be recovered by the observationally-induced approach. We restrict ourselves to ordinary nondeterminism (angelic, demonic and erratic) and compare our approach to the upper (Hoare), lower (Smyth) and convex (Plotkin) powerdomains. In these cases we are indeed in a position to describe the repletion explicitly which is a rare phenomenon. While in the angelic and demonic case we obtain the same result as classically, in the erratic case there is a big gap between the two approaches.

In a topological setting, the results for the Hoare and Smyth powerdomains have been worked out in [4, 5. The proof strategy is the same in the cases at hand, so we only sketch the proofs, as the reader should have no problems filling in the details by consulting loc. cit. The erratic case will be worked out in detail.

A nondeterministic (binary) choice operator $\forall$ is reasonably supposed to satisfy the following equational laws:

$$
\begin{aligned}
x \bullet x & =x & & \text { idempotency } \\
x \forall y & =y \forall x & & \text { commutativity } \\
x \forall(y \forall z) & =(x \forall y) \forall z & & \text { associativity }
\end{aligned}
$$

These laws characterize semilattices. A dcpo together with a continuous semilattice operation $\bullet$ is called a dcpo-semilattice.

One must be careful: In a dcpo-semilattice, $x \forall y$ need not be the least upper bound nor greatest lower bound of $x$ and $y$ with respect to the dcpo order $\leq$. A paradigmatic example is Heckmann's domain $\underline{\mathbb{A}}[10$, the three element chain

$$
\mathbb{A}=\{\perp<m<\top\}
$$

with the semilattice operation

$$
a \forall b= \begin{cases}a & \text { if } a=b \\ m & \text { else }\end{cases}
$$

where $T$ can be read as 'must', $m$ stands for 'may' and $\perp$ stands for 'impossible'. For constructing the Plotkin powerdomain in the observationally-induced approach, Simpson 23. has suggested to use Heckmann's domain $\mathbb{A}$ as computational prototype.

A dcpo-semilattice in which $x \forall y$ is the least upper bound of $x$ and $y$ will be called a dcpo-join-semilattice and, in this case, we will usually denote the semilattice operation by $\vee$. Among dcpo-semilattices the dcpo-join-semilattices are characterized by the inequational law

$$
x \leq x \cup y .
$$

A dcpo-semilattice in which $x \sqcup y$ is the greatest lower bound of $x$ and $y$ will be called a dcpomeet-semilattice2 2 and, in this case, we will usually denote the semilattice operation by $\wedge$. Among dcpo-semilattices the dcpo-meet-semilattices are characterized by the inequational law

$$
x \cup y \leq x .
$$

\footnotetext{
${ }^{2}$ Let us point out that our terminology deviates from the one mostly used in earlier texts, where the terms inflationary and deflationary are used for join- and meet-semilattices, respectively.
} 
The paradigmatic examples are obtained from the two element chain

$$
\Sigma=\{0<1\}
$$

by considering the operation $x \vee y=\max (x, y)$ in the first and the operation $x \wedge y=\min (x, y)$ in the second case. We will write

$$
\Sigma_{\vee}=(\Sigma, \vee) \text { and } \Sigma_{\wedge}=(\Sigma, \wedge)
$$

for the dcpo $\Sigma$ considered as a join-semilattice and meet-semilattice, respectively.

Semantically, dcpo-join-semilattices are modelling angelic nondeterminism: having a choice is considered to be preferable to an optimistic observer, since it opens the possibility to make the best choice; dcpo-meet-semilattices are modelling demonic nondeterminism: having a choice is considered to be undesirable to a pessimistic observer, since it opens the possibility to make the worst possible choice. General dcpo-semilattices are combining the angelic with the demonic point of view.

Indeed, if $\underline{A}$ is a dcpo-join-semilattice and $\underline{B}$ a dcpo-meet-semilattice, the direct product $\underline{A} \times \underline{B}$ with the operation $\left(x_{1}, y_{1}\right) \forall\left(x_{2}, y_{2}\right)=\left(x_{1} \vee x_{2}, y_{1} \wedge y_{2}\right)$ is a dcpo-semilattice which is neither a dcpo-join- nor a dcpo-meet-semilattice. Note that in particular $\mathbb{A}$ may be viewed as a subsemilattice of $\Sigma_{\vee} \times \Sigma_{\wedge}$ with the identifications $\perp=(0,0), m=(1,0)$ and $\top=(1,1)$.

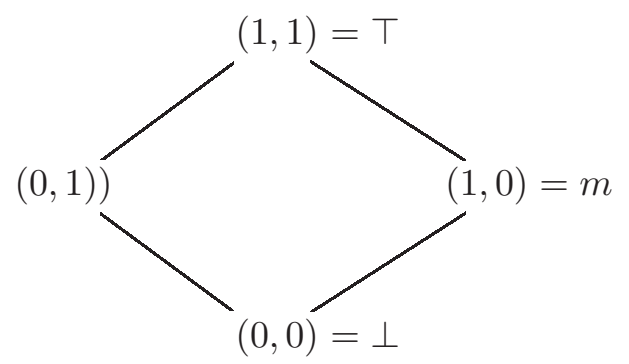

As one does not want to ask for any more than just the equational and inequational laws indicated above, the classical powerdomains over a dcpo $X$ are defined to be the free dcpo-semilattice $\mathcal{P}(X)$, the Plotkin powerdomain, the free dcpo-join-semilattice $\mathcal{H}(X)$, the Hoare powerdomain, and the free dcpo-meet-semilattice $\mathcal{S}(X)$, the Smyth powerdomain.

For applying the previously developed concepts to powerdomains, we place ourselves in the situation where the signature $\Omega$ consists of a single binary operation symbol. The $\mathcal{D}$ po $_{\Omega^{-}}$-algebras are simply dcpos with a continuous binary operation. The dcpo-semilattices form a full subcategory.

For the category of dcpo-semilattices we are in an exceptional situation: For two Scottcontinuous semilattice homomorphisms $f, f^{\prime}: \underline{A} \rightarrow \underline{B}$, we may define $f \forall f^{\prime}$ pointwise by $\left(f \cup f^{\prime}\right)(x)=f(x) \bullet f^{\prime}(x)$ for all $x \in \underline{A}$ and we see that $f \cup f^{\prime}$ is again a continuous semilattice homomorphism. Thus, the dcpo of all continuous semilattice homomorphisms $f: \underline{A} \rightarrow \underline{B}$ is a dcpo-subsemilattice of $\underline{B}^{A}$; we denote it by hom $(\underline{A}, \underline{B})$. If $\underline{B}$ has a bottom or a top element, the same holds for $\operatorname{hom}(\underline{A}, \underline{B})$ - consider the constant functions with value top and bottom, respectively. If $\underline{A}$ and $\underline{B}$ both have a bottom and a top element, we may restrict our attention to the dcpo-subsemilattice hom ${ }_{0,1}(\underline{A}, \underline{B})$ of all continuous semilattice homomorphisms that preserve bottom and top. We note that $\operatorname{hom}_{0,1}(\underline{A}, \underline{B})$ will not have a bottom or a top element, in general.

Lemma 4.1. For every dcpo-semilattice $\underline{R}$ with bottom and top and every dcpo $X$, the dcpo-semilattices hom $\left(\underline{R}^{X}, \underline{R}\right)$ and $\operatorname{hom}_{0,1}\left(\underline{R}^{X}, \underline{R}\right)$ are $\underline{R}$-complete. 
Indeed, $\underline{R}^{R^{X}}$ is $\underline{R}$-complete by Cor. 2.5 . The dcpo-subsemilattices $\operatorname{hom}(\underline{A}, \underline{B})$ and $\operatorname{hom}_{0,1}(\underline{A}, \underline{B})$ are carved out from $\underline{B}^{A}$ as intersections of equalizers of homomorphisms (which to check requires the associativity, commutativity and idempotence of $\uplus$ ). Thus $\operatorname{hom}(\underline{A}, \underline{B})$ and $\operatorname{hom}_{0,1}(\underline{A}, \underline{B})$ are $\underline{R}$-complete, since the category $\mathcal{D} c p o_{\underline{R}}$ of $\underline{R}$-complete algebras is limit closed by Lemma 1.5 .

Convention. For a dcpo $X$, we identify continuous maps $f: X \rightarrow \Sigma$ with the corresponding Scott-open subsets $U:=f^{-1}(\{1\}) \subseteq X$. In this way, the dcpo $\Sigma^{X}$ of continuous functions $u: X \rightarrow \Sigma$ with the pointwise order is identified with the dcpo $\mathcal{O}(X)$ of Scott-open subsets of $X$ ordered by inclusion.

4.1. Angelic nondeterminism. The prototype algebra for angelic nondeterminism is $\mathcal{H}(\mathbb{S})$, i.e. the algebra $\Sigma_{\vee}$. We will see that in this case the observationally-induced powerdomains agree with the classical Hoare powerdomains.

First we reproduce the standard representation of the Hoare powerdomain (see, e.g. [7]):

Proposition 4.2. The Hoare powerdomain over a dcpo $X$ is (isomorphic to) the dcpo $\mathcal{H}(X)$ of nonempty Scott-closed subsets of $X$ ordered by inclusion, the join-semilattice operation being binary union; the natural embedding $\eta_{X}^{H}: X \rightarrow \mathcal{H}(X)$ sends every $x \in X$ to its Scott closure $\downarrow x$.

Indeed, if $f$ is a continuous map from $X$ to some dcpo-join-semilattice $\underline{B}$, then $\widetilde{f}(C)=$ $\bigvee_{x \in C} f(x)$ is the unique continuous homomorphic extension of $f$ along $\eta^{H}$. From this definition of the extension it follows immediately that $f \leq f^{\prime}$ implies $\tilde{f} \leq \tilde{f}^{\prime}$. This implies that the Hoare powerdomain is internally free over $X$.

For our purposes we want to pass to a functional representation:

Lemma 4.3. The Hoare powerdomain over a dcpo $X$ is (isomorphic to) the dcpo-joinsemilattice $\operatorname{hom}_{0,1}\left(\Sigma_{\vee}^{X}, \Sigma_{\vee}\right)$ of all continuous semilattice homomorphism from $\Sigma_{\vee}^{X}$ to $\Sigma_{\vee}$ preserving bottom and top. The embedding $\eta_{X}^{H}: X \rightarrow \operatorname{hom}_{0,1}\left(\Sigma_{\vee}^{X}, \Sigma_{\vee}\right)$ is given by evaluation $\eta_{X}^{H}(x)(u)=u(x)$.

The isomorphism from the $\cup$-semilattice of nonempty closed subsets of $X$ to the function space $\operatorname{hom}_{0,1}\left(\Sigma_{\vee}^{X}, \Sigma_{\vee}\right)$ is given by assigning to every nonempty closed subset $C$ of $X$ the map $\varphi: \Sigma_{\vee}^{X} \rightarrow \Sigma_{\vee}$ defined by $\varphi(U)=0$ if and only if $U \cap C=\emptyset$. $\operatorname{hom}_{0,1}\left(\Sigma_{\vee}^{X}, \Sigma_{\vee}\right)$ is $\Sigma_{\vee}$-complete by Lemma 4.1; it is not only a dcpo-subsemilattice of $\Sigma_{V}^{\Sigma^{X}}$ but even the smallest dcpo-subsemilattice of $\Sigma_{\vee}^{\Sigma^{X}}$ containing the point evaluations $\eta_{X}^{H}(x), x \in X$, since $\operatorname{hom}_{0,1}\left(\Sigma_{\vee}^{X}, \Sigma_{\vee}\right)$ is the free dcpo-join-semilattice over $X$. Hence $\eta_{X}^{H}: X \rightarrow \operatorname{hom}_{0,1}\left(\Sigma_{\vee}^{X}, \Sigma_{\vee}\right)$ is the $\Sigma_{\bigvee}$-repletion of $X$. Altogether we have:

Theorem 4.4. The dcpo-join-semilattice $\operatorname{hom}_{0,1}\left(\Sigma_{\vee}^{X}, \Sigma_{\vee}\right)$ is the $\Sigma_{\vee}$-repletion of $X$. Up to isomorphism, the Hoare powerdomain $\eta_{X}^{H}: X \rightarrow \mathcal{H}(X)$ over a dcpo $X$ agrees with the $\Sigma_{\vee}$-repletion of $X$. 
4.2. Demonic nondeterminism. The prototype for demonic nondeterminism is $\mathcal{S}(\mathbb{S})$, i.e. the algebra $\Sigma_{\wedge}$.

There is no explicit description available for the free dcpo-meet-semilattice over an arbitrary dcpo $X$. However, for continuous dcpo's $X$ the free dcpo-meet-semilattice over $X$ will give rise to the classical Smyth powerdomain $\mathcal{S}(X)$.

The standard representation of the Smyth powerdomain over a continuous dcpo is the following ([7]):

Proposition 4.5. The Smyth powerdomain over a continuous dcpo $X$ is (isomorphic to) the dcpo $\mathcal{S}(X)$ of nonempty compact saturated subsets $Q$ of $X$ ordered by reverse inclusion $\supseteq$, the 'meet'-semilattice operation being binary union; the natural embedding $\eta_{X}^{S}: X \rightarrow \mathcal{S}(X)$ sends every $x \in X$ to its upper closure $\uparrow x$.

Indeed, if $f$ is a continuous map from a continuous dcpo $X$ to some dcpo-meet-semilattice $B$, its unique extension to a meet-semilattice homomorphism is given by $\widetilde{f}(Q)=$ $\bigvee_{F \in \mathcal{F}}^{\uparrow} \bigwedge_{x \in F} f(x)$, where $F$ ranges over the collection $\mathcal{F}$ of finite subsets $F$ of $X$ such that $Q$ is contained in the Scott-interior of $\uparrow F$. From the definition of the extension it follows directly that, if $f \leq f^{\prime}$, then $\widetilde{f} \leq \widetilde{f}^{\prime}$. Thus, the Smyth powerdomain over a continuous dcpo is internally free.

For any dcpo $X$, the dcpo-meet-semilattice $\operatorname{hom}_{0,1}\left(\Sigma_{\wedge}^{X}, \Sigma_{\wedge}\right)$ is isomorphic to the collection $\mathcal{O} \mathcal{F}(X)$ of all Scott-open (proper) filters of the dcpo $\mathcal{O}(X)$ of all Scott-open subsets of $X$ ordered by inclusion with binary intersection as semilattice operation. The isomorphism is given by assigning to $\varphi \in \operatorname{hom}_{0,1}\left(\Sigma_{\wedge}^{X}, \Sigma_{\wedge}\right)$ the inverse image $\varphi^{-1}(1)$.

If the dcpo $X$ is sober, $\mathcal{O F}(X)$ is isomorphic to the collection of all nonempty compact saturated subsets $Q$ of $X$ ordered by reverse inclusion $\supseteq$ with binary union as semilattice operation by [7, Theorem II-1.20]. The isomorphism is given by assigning to every $\mathcal{U} \in$ $\mathcal{O F}(X)$ its intersection $Q=\bigcap \mathcal{U}$. Since every continuous dcpo is sober, we have the functional representation:

Lemma 4.6. The Smyth powerdomain over a continuous dcpo $X$ is (isomorphic to) the dcpo-meet-semilattice $\operatorname{hom}_{0,1}\left(\Sigma_{\wedge}^{X}, \Sigma_{\wedge}\right)$ of all continuous semilattice homomorphism from $\Sigma_{\Lambda}^{X}$ to $\Sigma_{\wedge}$ preserving bottom and top. The embedding $\eta_{X}^{S}: X \rightarrow \operatorname{hom}_{0,1}\left(\Sigma_{\wedge}^{X}, \Sigma_{\wedge}\right)$ is given by evaluation $\eta_{X}^{S}(x)(u)=u(x)$.

By Lemma 4.1, hom ${ }_{0,1}\left(\Sigma_{\wedge}^{X}, \Sigma_{\wedge}\right)$ is $\Sigma_{\wedge}$-complete. If $X$ is continuous, then it is the free dcpo-meet-semilattice over $X$ by 4.6. Thus, there is no proper dcpo-meet-subsemilattice containing the evaluation maps $\eta_{X}^{S}(x), x \in X$. We conclude:

Theorem 4.7. For every continuous dcpo $X$, the dcpo-meet-semilattice $\operatorname{hom}_{0,1}\left(\Sigma_{\wedge}^{X}, \Sigma_{\wedge}\right)$ is the $\Sigma_{\wedge}$-repletion of $X$. Thus up to isomorphism, the Smyth powerdomain $\eta_{X}^{S}: X \rightarrow \mathcal{S}(X)$ over a continuous dcpo $X$ agrees with the $\Sigma_{\wedge}$-repletion of $X$.

4.3. Erratic nondeterminism. The prototype algebra for erratic nondeterminism is $\mathcal{P}(\mathbb{S})$, i.e. the algebra whose underlying dcpo is the three element chain $\mathbb{A}$ with the semilattice operation $\forall$ as defined at the beginning of this section.

Erratic nondeterminism seems to be much more complicated to handle than angelic or demonic nondeterminism. In contrast to the angelic and demonic case we arrive at the conclusion that the Plotkin powerdomain does not agree with $\mathbb{A}$-repletion; it is in fact 
properly contained in the repletion. The following theorem is expressing this claim. In this theorem we need the additional hypothesis that the dcpo is compact for its Scott topology. This is equivalent to the requirement that there is a finite subset $F$ such that every element is above some element in $F$. In particular, if the dcpo has a bottom element, then it is Scott-compact.

Theorem 4.8. (a) For a compact continuous dcpo $X$, the dcpo-semilattice $\operatorname{hom}_{0,1}\left(\mathbb{A}^{X}, \mathbb{A}\right)$ of all continuous semilattice homomorphisms $\varphi: \mathbb{A}^{X} \rightarrow \mathbb{A}$ preserving bottom and top is the $\mathbb{A}$-repletion of $X$, that is, $\operatorname{hom}_{0.1}\left(\mathbb{A}^{X}, \mathbb{A}\right)$ is the least $\mathbb{A}$-complete dcpo-subsemilattice of $\mathbb{A}^{\mathbb{A}^{X}}$ containing all the evaluation maps $\eta_{X}^{P}(x)=(u \mapsto u(x)), x \in X$.

(b) For a continuous dcpo $X$, the Plotkin power domain $\mathcal{P}(X)$ is (isomorphic to) the least dcpo-subsemilattice of $\mathbb{A}^{\mathbb{A}^{X}}$ containing all the evaluation maps $\eta_{X}^{P}(x)=(u \mapsto u(x)), x \in$ $X$.

Let us indicate, how big the gap between the Plotkin powerdomain and the repletion hom $_{0.1}\left(\mathbb{A}^{X}, \mathbb{A}\right)$ really is: As we will see in 4.3.2, the elements of the repletion of $X$ correspond to 'formal lenses', that is, pairs $(C, Q)$ of a closed set $C$ and a compact saturated set $Q$ in $X$ with nonempty intersection. Each of this formal lenses determines a 'real lens' $L=C \cap Q$. The same real lens can be represented by many different formal lenses. If the dcpo is continuous and coherent, the real lenses correspond to the elements of the Plotkin powerdomain as we recall later in 4.3.2.

A first step towards a proof of Theorem 4.8 is a consequence of Lemma 4.1 ,

Proposition 4.9. For any dcpo $X$, the dcpo-semilattice $\operatorname{hom}_{0,1}\left(\mathbb{A}^{X}, \mathbb{A}\right)$ of all top and bottom preserving semilattice homomorphisms $\varphi: \mathbb{A}^{X} \rightarrow \mathbb{A}$ is $\mathbb{A}$-complete.

For $\eta_{X}^{P}: X \rightarrow \operatorname{hom}_{0,1}\left(\mathbb{A}^{X}, \mathbb{A}\right)$ being the $\mathbb{A}$-repletion of $X$, it suffices to show now that $\eta_{X}^{P}$ is $\mathbb{A}$-equable, i.e. that for every continuous $f: X \rightarrow \mathbb{A}$ there exists a unique Scott-continuous homomorphism $\widehat{f}: \operatorname{hom}_{0,1}\left(\mathbb{A}^{X}, \mathbb{A}\right) \rightarrow \mathbb{A}$ with $\widehat{f} \circ \eta_{X}^{P}=f$ and $f \mapsto \widehat{f}$ is continuous. Actually, existence and continuity of $\widehat{f}$ is not the problem since one may take $\widehat{f}(\varphi)=\varphi(f)$. The question rather is uniqueness. For answering this question positively we prove an auxiliary result.

\subsubsection{An auxiliary observation.}

(1) Let $A_{1}$ and $B_{1}$ be dcpo-join-semilattices and $A_{2}$ and $B_{2}$ dcpo-meet-semilattices.

The direct product $A_{1} \times A_{2}$ (and similarly $B_{1} \times B_{2}$ ) is a dcpo-semilattice with the product order

$$
\left(a_{1}, a_{2}\right) \leq\left(a_{1}^{\prime}, a_{2}^{\prime}\right) \Longleftrightarrow a_{1} \leq a_{1}^{\prime}, a_{2} \leq a_{2}^{\prime}
$$

and the semilattice operation

$$
\left(a_{1}, a_{2}\right) \bullet\left(a_{1}^{\prime}, a_{2}^{\prime}\right)=\left(a_{1} \vee a_{1}^{\prime}, a_{2} \wedge a_{2}^{\prime}\right) .
$$

We denote by $\pi_{i}: A_{1} \times A_{2} \rightarrow A_{i}, i=1,2$, the canonical projections onto the factors. We use the same notation for the projections of $B_{1} \times B_{2}$ onto its factors.

(2) We suppose in addition that $B_{1}$ has a top element 1 and $B_{2}$ a bottom element 0 .

Then we have embeddings $\varepsilon_{i}: B_{i} \rightarrow B_{1} \times B_{2}$ defined by $\varepsilon_{1}\left(b_{1}\right)=\left(b_{1}, 0\right)$ and $\varepsilon_{2}\left(b_{2}\right)=\left(1, b_{2}\right)$, respectively, which are semilattice homomorphisms.

(3) Suppose that $B$ is a dcpo-subsemilattice of $B_{1} \times B_{2}$ containing $\left(b_{1}, 0\right)$ and $\left(1, b_{2}\right)$ for all $b_{1} \in B_{1}, b_{2} \in B_{2}$. 


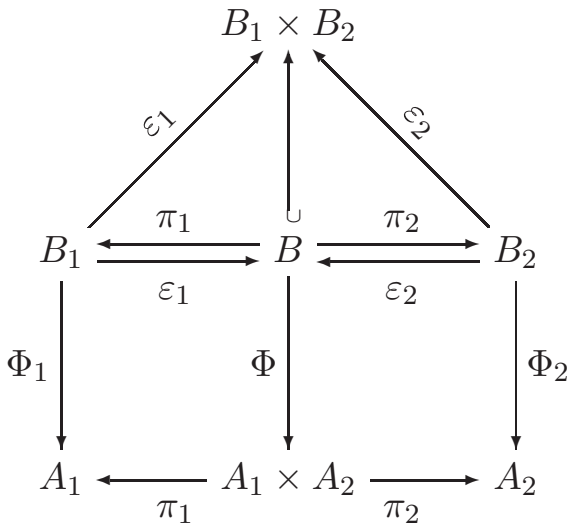

Observation 4.10. For a continuous semilattice homomorphism $\Phi: B \rightarrow A_{1} \times A_{2}$ consider the continuous semilattice homomorphisms $\Phi_{i}=\pi_{i} \Phi \varepsilon_{i}: B_{i} \rightarrow A_{i}, i=1,2$, that is, $\Phi_{1}\left(b_{1}\right)=$ $\pi_{1}\left(\Phi\left(b_{1}, 0\right)\right)$ and $\Phi_{2}\left(b_{2}\right)=\pi_{2}\left(\Phi\left(1, b_{2}\right)\right)$ for all $b_{1} \in B_{1}, b_{2} \in B_{2}$. Then the above diagram commutes, that is, $\Phi\left(b_{1}, b_{2}\right)=\left(\Phi_{1}\left(b_{1}\right), \Phi_{2}\left(b_{2}\right)\right)$ for all $b=\left(b_{1}, b_{2}\right) \in B$.

Proof. Let $\Phi: B \rightarrow A_{1} \times A_{2}$ be a semilattice homomorphism. The composed maps $\psi_{i}=$ $\pi_{i} \Phi: B \rightarrow A_{i}$ are semilattice homomorphisms, too. We claim that $\psi_{1}$ does not depend on the second and $\psi_{2}$ not on the first argument.

Indeed, for $\left(b_{1}, b_{2}\right),\left(b_{1}, b_{2}^{\prime}\right) \in B$ we have: $\psi_{1}\left(b_{1}, b_{2}\right), \psi_{1}\left(b_{1}, b_{2}^{\prime}\right) \leq \psi_{1}\left(b_{1}, b_{2}\right) \vee \psi_{1}\left(b_{1}, b_{2}^{\prime}\right)=$ $\psi_{1}\left(\left(b_{1}, b_{2}\right) \forall\left(b_{1}, b_{2}^{\prime}\right)\right)=\psi_{1}\left(b_{1}, b_{2} \wedge b_{2}^{\prime}\right) \leq \psi_{1}\left(b_{1}, b_{2}\right), \psi_{1}\left(b_{1}, b_{2}^{\prime}\right)$. Thus $\psi_{1}\left(b_{1}, b_{2}\right)=\psi_{1}\left(b_{1}, b_{2}^{\prime}\right)$. The order dual argument yields the analogous statement for $\psi_{2}$.

It follows that the maps $\Phi_{i}: B_{i}: \rightarrow A_{i}, i=1,2$, defined by $\Phi_{1}\left(b_{1}\right)=\psi_{1}\left(b_{1}, 0\right)=$ $\pi_{1} \Phi\left(b_{1}, 0\right)$ and $\Phi_{2}\left(b_{2}\right)=\psi_{2}\left(1, b_{2}\right)=\pi_{2} \Phi\left(1, b_{2}\right)$ have the desired properties.

4.3.2. Formal lenses and real lenses. Our aim in this subsection is to develop a more conceptual understanding of the elements of $\operatorname{hom}_{0,1}\left(\mathbb{A}^{X}, \mathbb{A}\right)$ as so-called formal lenses in a dcpo $X$.

Recall that $\mathbb{A}$ can be seen as the subsemilattice of $\Sigma_{\vee} \times \Sigma_{\wedge}$ consisting of those elements $\left(a_{1}, a_{2}\right)$ with $a_{1} \geq a_{2}$.

Consequently, every $f \in \mathbb{A}^{X}$ can be written uniquely as a pair $\left(u_{1}, u_{2}\right) \in \Sigma_{\vee}^{X} \times \Sigma_{\wedge}^{X}$ such that $u_{1} \geq u_{2}$ or, alternatively, as a pair $\left(U_{1}, U_{2}\right)$ of open subsets of $X$ with $U_{1} \supseteq U_{2}$.

Lemma 4.11. hom $_{0,1}\left(\mathbb{A}^{X}, \mathbb{A}\right)$ agrees with the dcpo-subsemilattice of all pairs $\left(\varphi_{1}, \varphi_{2}\right)$ in $\operatorname{hom}_{0,1}\left(\Sigma_{\vee}^{X}, \Sigma_{\vee}\right) \times \operatorname{hom}_{0,1}\left(\Sigma_{\wedge}^{X}, \Sigma_{\wedge}\right)$ such that $\varphi_{1} \geq \varphi_{2}$.

Proof. We want to apply Observation 4.10, $\mathbb{A}^{X}$ is the dcpo-subsemilattice of $\Sigma_{\vee}^{X} \times \Sigma_{\Lambda}^{X}$ of all $u=\left(u_{1}, u_{2}\right)$ such that $u_{1} \geq u_{2}$. In particular $\left(u_{1}, 0\right) \in \mathbb{A}^{X}$ and $\left(1, u_{2}\right) \in \mathbb{A}^{X}$ for all $u_{1}, u_{2} \in \Sigma^{X}$, where 0 and 1 denote also the constant functions with value 0 and 1 respectively. Thus the hypotheses of 4.10 are satisfied. We conclude that the continuous semilattice homomorphisms $\varphi: \mathbb{A}^{X} \rightarrow \Sigma_{\vee} \times \Sigma_{\wedge}$ are those maps for which there are continuous semilattice homomorphisms $\varphi_{1}: \Sigma_{\vee}^{X} \rightarrow \Sigma_{\vee}$ and $\varphi_{2}: \Sigma_{\wedge}^{X} \rightarrow \Sigma_{\wedge}$ such that $\varphi\left(u_{1}, u_{2}\right)=\left(\varphi_{1}\left(u_{1}\right), \varphi_{2}\left(u_{2}\right)\right)$. The maps $\varphi$ and $\varphi_{1}, \varphi_{2}$ are related by the formulas $\varphi_{1}\left(u_{1}\right)=\pi_{1} \varphi\left(u_{1}, 0\right)$ and $\varphi_{2}\left(u_{2}\right)=\pi_{2} \varphi\left(1, u_{2}\right)$. Clearly, $\varphi$ preserves bottom and top if and only if both $\varphi_{1}$ and $\varphi_{2}$ preserve bottom and top. Finally, $\varphi$ maps $\mathbb{A}^{X}$ into $\mathbb{A}$ if and only if $\varphi_{1} \geq \varphi_{2}$. 
According to Lemma 4.3 and Lemma 4.6, the elements $\varphi_{1}$ of $\operatorname{hom}_{0,1}\left(\Sigma_{\vee}^{X}, \Sigma_{\vee}\right)$ correspond to nonempty closed subsets $C$ of $X$, namely $C=X \backslash \cup \varphi_{1}^{-1}(0)$, and the elements $\varphi_{2}$ of $\operatorname{hom}_{0,1}\left(\Sigma_{\wedge}^{X}, \Sigma_{\wedge}\right)$ correspond to Scott-open filters $\mathcal{U}$ of $\mathcal{O}(X)$, namely $\mathcal{U}=\varphi_{2}^{-1}(1)$. The condition $\varphi_{1} \geq \varphi_{2}$ corresponds to the requirement $C \cap U \neq \emptyset$ for all $U \in \mathcal{U}$. Hence $\operatorname{hom}_{0,1}\left(\mathbb{A}^{X}, \mathbb{A}\right)$ is the functional representation of the dcpo-semilattice of formal lenses according to the following definition:

Definition 4.12. A formal lens of a dcpo $X$ consists of a pair $(C, \mathcal{U})$ of a nonempty closed subset $C$ of $X$ and a Scott-open filter $\mathcal{U}$ of $\mathcal{O}(X)$ such that $C \cap U \neq \emptyset$ for all $U \in \mathcal{U}$.

The formal lenses form a dcpo-subsemilattice of $\mathcal{C}(X) \times \mathcal{O} \mathcal{F}\rangle \uparrow \sqcup(X)$, where $\mathcal{C}(X)$ is the dcpo- $\cup$-semilattice of nonempty closed subsets of $X$ and $\mathcal{O} \mathcal{F}\rangle \downarrow \sqcup(X)$ is the dcpo- $\cap$ semilattice of Scott-open filters of $\mathcal{O}(X)$.

In the case where the dcpo $X$ is sober w.r.t. its Scott topology, under assumption of AC the Scott-open filters $\mathcal{U}$ can be replaced by their intersections $Q=\bigcap \mathcal{U}$ which are the nonempty Scott-compact saturated subsets of $X$. Thus the formal lenses of a sober dcpo $X$ are the pairs $(C, Q)$ of closed subsets $C$ and compact saturated subsets $Q$ of $X$ such that $C \cap Q \neq \emptyset$. But remember that the dcpo of nonempty compact saturated sets is ordered by reverse inclusion $\supseteq$ and the meet-operation is $\cup$.

Notice that formal lenses $(C, Q)$ are not determined by the intersection $L:=C \cap Q$ which can be considered as the real lens corresponding to the formal lens $(C, Q)$. This real lens $L$ can be represented by the "normalized" formal lens $\left(C_{L}, Q_{L}\right)$ where $C_{L}$ is the closure of $L$ for the Scott topology and $Q_{L}=\uparrow L$.

In most cases the dcpo of formal lenses is much bigger than the dcpo of real lenses. As an example consider the two element set $X=\{0,1\}$ with the discrete order. There are three real lenses, the nonempty subsets. But there are four more formal lenses. The real lens $\{1\}$ has three representations by formal lenses, namely $\{1\}=\{1\} \cap\{1\}=\{1\} \cap\{0,1\}=$ $\{0,1\} \cap\{1\}$, and so has $\{0\}$. Thus, for flat dcpos $X$ with more than one element $\mathbb{A}(X)$ is different from the Plotkin powerdomain $\mathcal{P}(X)$ and the same applies to their liftings $X_{\perp}$.

4.3.3. The $\mathbb{A}$-repletion of a compact continuous dcpo. Since $\operatorname{hom}_{0,1}\left(\mathbb{A}^{X}, \mathbb{A}\right)$ is $\mathbb{A}$-complete by Proposition 4.9 , the question now is whether the map $\eta^{P}: X \rightarrow \operatorname{hom}_{0,1}\left(\mathbb{A}^{X}, \mathbb{A}\right): x \mapsto$ $\lambda \varphi . \varphi(x)$ is $\mathbb{A}$-equable. I. Battenfeld in his 2013 paper has shown that this is actually true for $X=2$, the discrete poset. In this section we give a positive answer for compact continuous depos $X$.

As before, we consider $\mathbb{A}$ to be a dcpo-subsemilattice of $\Sigma_{\vee} \times \Sigma_{\wedge}$. We write $\pi_{1}: \Sigma_{\vee} \times$ $\Sigma_{\wedge} \rightarrow \Sigma_{\vee}$ and $\pi_{2}: \Sigma_{\vee} \times \Sigma_{\wedge} \rightarrow \Sigma_{\wedge}$ for the respective canonical projections.

By Lemma 4.11, hom ho, $\left(\mathbb{A}^{X}, \mathbb{A}\right)$ agrees with the dcpo-subsemilattice of all $\left(\varphi_{1}, \varphi_{2}\right) \in$ $\operatorname{hom}_{0,1}\left(\Sigma_{\vee}^{X}, \Sigma_{\vee}\right) \times \operatorname{hom}_{0,1}\left(\Sigma_{\wedge}^{X}, \Sigma_{\wedge}\right)$ such that $\varphi_{1} \geq \varphi_{2}$. We write $\pi_{H}$ and $\pi_{S}$ for the projections from $\operatorname{hom}_{0,1}\left(\mathbb{A}^{X}, \mathbb{A}\right)$ to $\operatorname{hom}_{0,1}\left(\Sigma_{\vee}^{X}, \Sigma_{\vee}\right)$ and $\operatorname{hom}_{0,1}\left(\Sigma_{\wedge}^{X}, \Sigma_{\wedge}\right)$, respectively. The maps $\eta^{P}: X \rightarrow \operatorname{hom}_{0,1}\left(\mathbb{A}^{X}, \mathbb{A}\right), \eta^{H}: X \rightarrow \operatorname{hom}_{0,1}\left(\Sigma_{\vee}^{X}, \Sigma_{\vee}\right)$ and $\eta^{S}: X \rightarrow \operatorname{hom}_{0,1}\left(\Sigma_{\wedge}^{X}, \Sigma_{\wedge}\right)$ are all 
three given by $\lambda x . \lambda u . u(x)$ as usual and one easily checks that the diagram

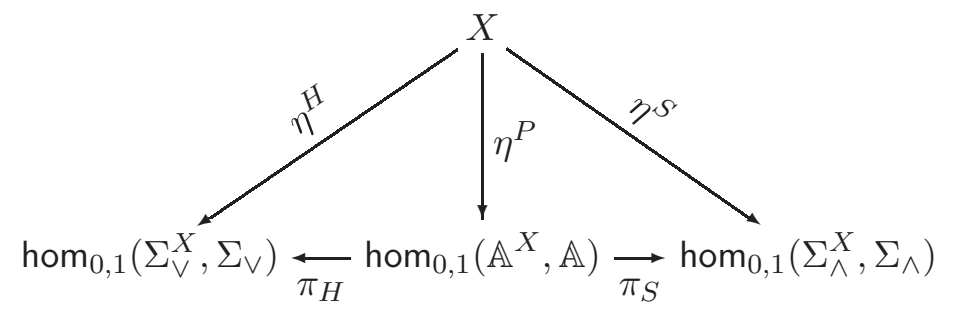

commutes. In order to apply Observation 4.10, we need the hypothesis that hom ${ }_{0,1}\left(\Sigma_{\wedge}^{X}, \Sigma_{\wedge}\right)$ has a least and $\operatorname{hom}_{0,1}\left(\Sigma_{\vee}^{X}, \Sigma_{\vee}\right)$ a greatest element. While hom ${ }_{0,1}\left(\Sigma_{\vee}^{X}, \Sigma_{\vee}\right)$ always has a greatest element - the map $\varphi_{\top}$ that maps all open sets to top except for the empty set, we have to suppose $X$ to be compact for the Scott topology so that hom ${ }_{0,1}\left(\Sigma_{\wedge}^{X}, \Sigma_{\wedge}\right)$ has a least element. Indeed, the map $\varphi_{\perp}$ mapping all open sets to bottom except for the whole space is a meet-semilattice homomorphism, and it is continuous if and only if $X$ is compact. Thus, we assume $X$ to be Scott-compact. We now have continuous maps $\varepsilon_{H}: \operatorname{hom}_{0,1}\left(\Sigma_{\vee}^{X}, \Sigma_{\vee}\right) \rightarrow \operatorname{hom}_{0,1}\left(\mathbb{A}^{X}, \mathbb{A}\right): \varphi_{1} \mapsto\left(\varphi_{1}, \varphi_{\perp}\right)$ with $\pi_{H} \circ \varepsilon_{H}=$ id, and $\varepsilon_{S}: \operatorname{hom}_{0,1}\left(\Sigma_{\wedge}^{X}, \Sigma_{\wedge}\right) \rightarrow \operatorname{hom}_{0,1}\left(\mathbb{A}^{X}, \mathbb{A}\right): \varphi_{2} \mapsto\left(\varphi_{\top}, \varphi_{2}\right)$ with $\pi_{S} \circ \varepsilon_{S}=$ id.

Now we can apply the decomposition Observation 4.10 and we obtain:

Lemma 4.13. Let $X$ be a compact dcpo and $\Phi: \operatorname{hom}_{0,1}\left(\mathbb{A}^{X}, \mathbb{A}\right) \rightarrow \mathbb{A}$ a continuous semilattice homomorphism. Then there exist unique continuous semilattice homomorphisms $\Phi_{H}: \operatorname{hom}_{0,1}\left(\Sigma_{\vee}^{X}, \Sigma_{\vee}\right) \rightarrow \Sigma_{\vee}$ and $\Phi_{S}: \operatorname{hom}_{0,1}\left(\Sigma_{\wedge}^{X}, \Sigma_{\wedge}\right) \rightarrow \Sigma_{\wedge}$ such that the following diagram commutes:

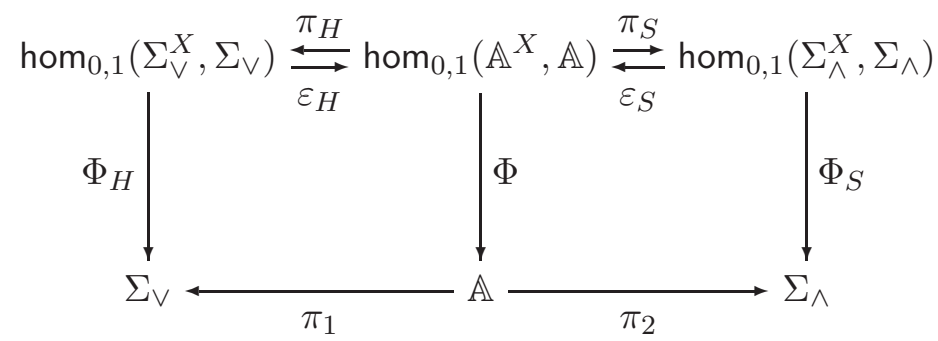

Now we are ready to show the result that we were aiming for.

Proposition 4.14. For compact continuous dcpos $X$ the map $\eta_{X}^{P}: X \rightarrow \operatorname{hom}_{0,1}\left(\mathbb{A}^{X}, \mathbb{A}\right)$ is A-equable.

Proof. Given $u: X \rightarrow \mathbb{A}$, a continuous homomorphic extension $\Phi: \operatorname{hom}_{0,1}\left(\mathbb{A}^{X}, \mathbb{A}\right) \rightarrow \mathbb{A}$ of $u$ along $\eta_{X}^{P}$ is given by $\Phi(\varphi)=\varphi(u)$. Clearly, $\Phi$ is a continuous semilattice homomorphism and we have $\Phi\left(\eta_{X}^{P}(x)\right)=\eta_{X}^{P}(x)(u)=u(x)$ for all $x \in X$, that is, $\Phi$ extends $u$ along $\eta_{X}^{P}$. The extension operator $u \mapsto \Phi$ is continuous since it is $\lambda$-definable as $\lambda u \cdot \lambda \varphi \cdot \varphi(u)$.

From Lemma 4.13 it follows that $\Phi$ is uniquely determined by the semilattice homomorphisms $\Phi_{H}$ and $\Phi_{S}$. We have $\Phi_{H} \circ \eta_{X}^{H}=\pi_{1} \circ u$ and $\Phi_{S} \circ \eta_{X}^{S}=\pi_{2} \circ u$. Since $\eta_{X}^{H}: X \rightarrow \mathcal{H}(X)$ is internally free it is also $\Sigma_{\vee}$-equable. Since $\eta_{X}^{S}: X \rightarrow \mathcal{S}(X)$ is internally free it is also

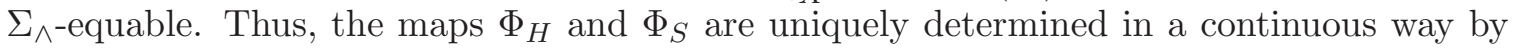
$\pi_{1} \circ u$ and $\pi_{2} \circ u$, respectively. Thus, the map $\Phi$ is uniquely determined by $u$ in a continuous way. 
The previous proposition together with Proposition 4.9 finishes the proof of part (a) of our main theorem 4.8

4.3.4. The Plotkin powerdomain and $\mathbb{A}$-valuations. We proceed to a proof of assertion (b) in our Main Theorem 4.8, We could give a direct proof. But we prefer to use R. Heckmann's work in 10 on $\mathbb{A}$-valuations and the Plotkin powerdomain.

Heckmann has called a continuous map $\alpha: \Sigma^{X} \rightarrow \mathbb{A}$ an $\mathbb{A}$-valuation on the dcpo $X$, if it preserves bottom and top and, moreover, satisfies the following two conditions:

(H1) if $\alpha(U)=\perp$ then $\alpha(U \cup V)=\alpha(V)$,

(H2) if $\alpha(U)=\top$ then $\alpha(U \cap V)=\alpha(V)$.

The collection H-Aval $(X)$ of Heckmann's $\mathbb{A}$-valuations is a dcpo-subsemilattice of $\mathbb{A}^{\Sigma^{X}}$.

We want to adapt Heckmann's $\mathbb{A}$-valuations to our setting. We can identify the semilattice $\mathrm{H}$-A val of Heckmann's $\mathbb{A}$-valuations with a dcpo-subsemilattice of $\operatorname{hom}_{0,1}\left(\mathbb{A}^{X}, \mathbb{A}\right)$. For this purpose we consider $\mathbb{A}$ as a join- and as a meet-semilattice with the operations

$$
a \vee b=\max (a, b), \quad a \wedge b=\min (a, b)
$$

where max and min refer do the dcpo-ordering of $\mathbb{A}$.

Definition 4.15. A continuous map $\varphi: \mathbb{A}^{X} \rightarrow \mathbb{A}$ will be called an $\mathbb{A}$-valuation on the dcpo $X$, if it preserves 0 and 1 and satisfies the conditions

(H1) if $\varphi(u)=\perp$ then $\varphi(u \vee v)=\varphi(v)$,

(H2) if $\varphi(u)=\top$ then $\varphi(u \wedge v)=\varphi(v)$.

We denote by $\mathbb{A} \operatorname{val}(X)$ the collection of these $\mathbb{A}$-valuations.

An easy calculation shows that one can pass from Heckmann's $\mathbb{A}$-valuations $\alpha$ to our $\mathbb{A}$-valuations by defining $\bar{\alpha}\left(U_{1}, U_{2}\right)=\alpha\left(U_{1}\right) \forall \alpha\left(U_{2}\right)$. Conversely, from an $\mathbb{A}$-valuation $\varphi$ in our sense on obtains an $\mathbb{A}$-valuation in the sense of Heckmann by defining $\bar{\varphi}(U)=\varphi(U, U)$. (Here we have used that the elements of $\mathbb{A}^{X}$ can be represented as pairs of open sets $u=\left(U_{1}, U_{2}\right)$ with $U_{1} \supseteq U_{2}$.)

For working with $\mathbb{A}$-valuations in our sense, another characterization is useful: As before, we consider $\mathbb{A}$ to be a subsemilattice of $\Sigma_{\vee} \times \Sigma_{\wedge}$ and we denote by $\pi_{i}, i=1,2$, the canonical projections onto the two factors. Similarly, we represent $\operatorname{hom}_{0,1}\left(\mathbb{A}^{X}, \mathbb{A}\right)$ as the dcpo-subsemilattice of all $\varphi=\left(\varphi_{1}, \varphi_{2}\right) \in \operatorname{hom}_{0,1}\left(\Sigma_{\vee}^{X}, \Sigma_{\vee}\right) \times \operatorname{hom}_{0,1}\left(\Sigma_{\wedge}^{X}, \Sigma_{\wedge}\right)$ such that $\varphi_{1} \geq \varphi_{2}$.

Lemma 4.16. Let $X$ be any dcpo.

(a) For every Heckmann $\mathbb{A}$-valuation $\alpha: \Sigma^{X} \rightarrow \mathbb{A}$, let $\bar{\alpha}_{i}=\pi_{i} \circ \alpha: \Sigma^{X} \rightarrow \Sigma, i=1,2$, and $\bar{\alpha}=\left(\bar{\alpha}_{1}, \bar{\alpha}_{2}\right)$. Then $\bar{\alpha} \in \operatorname{hom}_{0,1}\left(\mathbb{A}^{X}, \mathbb{A}\right)$.

(b) $A \varphi=\left(\varphi_{1}, \varphi_{2}\right) \in \operatorname{hom}_{0,1}\left(\mathbb{A}^{X}, \mathbb{A}\right)$ is of the form $\bar{\alpha}$ for some Heckmann $\mathbb{A}$-valuation $\alpha$ if and only if it satisfies the following two conditions:

(H1') if $\varphi_{1}(U)=\perp$ then $\varphi_{2}(V)=\varphi_{2}(U \cup V)$

(H2') if $\varphi_{2}(U)=\top$ then $\varphi_{1}(V)=\varphi_{1}(U \cap V)$

(c) The elements $\varphi=\left(\varphi_{1}, \varphi_{2}\right) \in \operatorname{hom}_{0,1}\left(\mathbb{A}^{X}, \mathbb{A}\right)$ satisfying (H1') and (H2') are exactly the $\mathbb{A}$-valuations according to the definition above. They form a dcpo-subsemilattice of $\operatorname{hom}_{0,1}\left(\mathbb{A}^{X}, \mathbb{A}\right)$ and $\alpha \mapsto \bar{\alpha}$ is a dcpo-semilattice isomorphism of $\mathrm{H}$-Aval onto $\mathbb{A} v a l(X)$. 
Proof. (a) It is clear that the $\alpha_{i}$ are continuous and that they preserve bottom and top. We check that $\alpha_{1}$ is a join-semilattice homomorphism. It suffices to show: if $\alpha_{1}(U)=\alpha_{1}(V)=0$ then $\alpha_{1}(U \cup V)=0$, and this is a direct consequence of Heckmann's condition (H1). One uses condition (H2) in a similar way to show that $\alpha_{2}$ is a meet-semilattice homomorphism. Since $\pi_{1} \geq \pi_{2}$, we also have $\alpha_{1}=\pi_{1} \circ \alpha \geq \pi_{2} \circ \alpha \geq \alpha_{2}$.

(b) For every Heckmann $\mathbb{A}$-valuation $\alpha, \alpha_{1}$ and $\alpha_{2}$ satisfy the conditions (H1') and (H2'). Indeed, if $\alpha_{1}(U)=\pi_{1}(\alpha(U))=0$, then $\alpha(U)=0$, whence $\alpha(U \cup V)=\alpha(V)$ by condition (H1); thus, $\alpha_{2}(U \cup V)=\pi_{2} \alpha(U \cup V)=\pi_{2} \alpha(U)=\alpha_{2}(V)$, which proves condition (H1'). Similarly one proceeds for (H2').

Conversely, for every $\varphi=\left(\varphi_{1}, \varphi_{2}\right) \in \operatorname{hom}_{0,1}\left(\mathbb{A}^{X}, \mathbb{A}\right)$ we define a map $\bar{\varphi}: \Sigma^{X} \rightarrow \mathbb{A}$ by $\bar{\varphi}(U)=\left(\varphi_{1}(U), \varphi_{2}(U)\right)$. Clearly $\bar{\varphi}$ is continuous and preserves bottom and top. If $\varphi$ satisfies (H1') and (H2'), then $\bar{\varphi}$ is a Heckmann $\mathbb{A}$-valuation. Indeed, suppose $\bar{\varphi}(U)=\perp$. Then $\left(\varphi_{1}(U), \varphi_{2}(U)\right)=\perp=(0,0)$. Thus, firstly, $\varphi_{1}(U)=0$ which implies that $\varphi_{1}(U \cup V)=$ $\varphi_{1}(U) \vee \varphi_{1}(V)=\varphi_{1}(V)$, where we have used that $\varphi_{1}$ is a join-homomorphism. Secondly, $\varphi_{1}(U)=0$ also implies $\varphi_{2}(U \cup V)=\varphi_{2}(V)$ by condition (H1'). Thus $\bar{\varphi}(U \cup V)=\left(\varphi_{1}(U \cup\right.$ $\left.V), \varphi_{2}(U \cup V)\right)=\left(\varphi_{1}(V), \varphi_{2}(V)\right)=\bar{\varphi}(V)$. Thus $\bar{\varphi}$ satisfies (H1). Similarly, $\bar{\varphi}$ satisfies (H2).

(c) Clearly $\overline{\bar{\alpha}}=\alpha$ and $\overline{\bar{\varphi}}=\varphi$. Thus, $\alpha \mapsto \bar{\alpha}$ and $\varphi \mapsto \bar{\varphi}$ are mutually inverse bijections. These bijections are order preserving by their very definition. Hence they are dcpo-isomorphisms. It is immediate from the definition that $\alpha \mapsto \bar{\alpha}$ is a semilattice homomorphism from $\mathbb{A} v a l(X)$ to $\operatorname{hom}_{0,1}\left(\mathbb{A}^{X}, \mathbb{A}\right)$.

There is a natural map $\delta: X \rightarrow \mathrm{H}$-Aval $(X)$ which to every $x \in X$ assigns the map $\delta(x): \Sigma^{X} \rightarrow \mathbb{A}$ defined as follows:

$$
\delta(x)(U)= \begin{cases}\top & \text { if } x \in U, \\ \perp & \text { else } .\end{cases}
$$

Clearly, $\delta(x)$ is a Heckmann $\mathbb{A}$-valuation and $\delta$ depends continuously on $x$. For every nonempty finite subset $F$ of $X$, we can form the $\mathbb{A}$-valuation $\delta(F)=\bigcup_{x \in F} \delta(x)$ which can be defined by

$$
\delta(F)(U)= \begin{cases}\top & \text { if } F \subseteq U, \\ \perp & \text { if } F \cap U=\emptyset, \\ m & \text { else. }\end{cases}
$$

Moreover, the following diagram commutes:

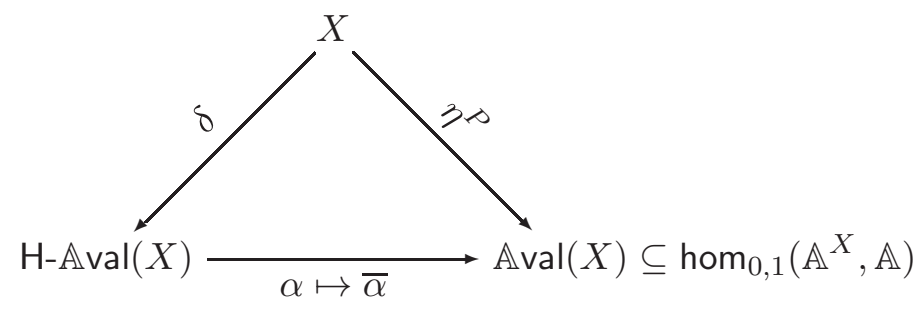

For continuous dcpos Heckmann has shown [10, Theorem 6.1] that $\mathrm{H}-\mathbb{A}$ val $(X)$ is (isomorphic to) the Plotkin powerdomain over $X$ and that the $\delta(F)$ for nonempty finite subsets $F$ of $X$ form a basis. Using the preceding commuting diagram we have:

Theorem 4.17. For any continuous dcpo $X$, the dcpo-semilattice $\mathbb{A}$ val $(X)$ of $\mathbb{A}$-valuations is (isomorphic to) the Plotkin powerdomain $\mathcal{P}(X)$, the canonical map being $\eta^{P}: X \rightarrow$ 
$\mathbb{A}$ val $(X)$. Moreover, $\mathbb{A} v a l(X)$ is a continuous dcpo. The $\mathbb{A}$-valuations $\eta^{P}(F)$ form a basis, when $F$ ranges over the nonempty finite subsets of $X$.

We now can finish the proof of part (b) of Theorem 4.8,

Proposition 4.18. For a continuous dcpo $X$, the Plotkin powerdomain is (isomorphic to) the least dcpo-subsemilattice of $\mathbb{A}^{\mathbb{A}^{X}}$ containing all the point evaluations $\eta_{X}^{P}(x)=\lambda u . u(x)$, $x \in X$.

Proof. From Heckmann's Theorem 4.17 we know that H-Aval $(X)$ is the free dcpo-semilattice over $X$. Thus $\mathrm{H}$-Aval $(X)$ has no proper dcpo-subsemilattice containing all the $\delta(x), x \in$ $X$. By the isomorphism established in the preceding lemma, there is a dcpo-semilattice isomorphism from $\mathrm{H}$-Aval $(X)$ onto the dcpo-subsemilattice $\mathbb{A}$ val $(X)$ of $\mathbb{A}^{\mathbb{A}^{X}}$ mapping $\delta(x)$ to the evaluation map $\eta_{X}^{P}(x), x \in X$. Thus this subsemilattice is the least dcpo-subsemilattice of $\mathbb{A}^{\mathbb{A}^{X}}$ containing all the $\eta_{X}^{P}(x), x \in X$.

Problem 4.19. In this context it seems appropriate to recall Birkhoff's theorem [6, pages 143f.] from universal algebra (in the category of sets): For a finitary signature $\Omega$, consider an $\Omega$-algebra $\underline{R}$. For any set $X$ let $\eta: X \rightarrow \underline{R}^{X}$ denote the canonical map defined by $\eta(x)(f)=f(x)$ for $x \in X$ and $f: X \rightarrow R$. Then the $\Omega$-subalgebra $F(X)$ of the product algebra $\underline{R}^{\underline{R}}$ generated by the 'projections' $\eta(x), x \in X$, is free in the equational class of $\Omega$-algebras satisfying all the equational laws that hold in $\underline{R}$.

One would like to transpose this result into the context of $\mathcal{D} c p o_{\Omega^{-a l g e b r a s}}$. Our last proposition seems to indicate that this is not an hopeless effort. Instead of equational laws one will also have to consider inequational laws, too. Thus one can ask the question: Given a $\mathcal{D}$ cpo ${ }_{\Omega}$-algebra $\underline{R}$ and a dcpo $X$. Is the least $\mathcal{D}_{\text {cpo }}{ }_{\Omega^{-}}$-subalgebra of $\underline{R}^{R^{X}}$ containing all the evaluation maps $\eta(x)=\lambda u$. u(x) free in the class of Dcpo $_{\Omega^{-}}$-algebras satisfying all equational and inequational laws that hold in $\underline{R}$ ?

Remark 4.20. We have seen that, in general, $\mathbb{A}$-valuations are more general than lenses. In order to describe $\mathbb{A}$-valuations in general, J. Goubault-Larrecq [9] has introduced the notion of a quasilens. This is a formal lens $(C, \mathcal{U})$ where $C$ is a nonempty closed set and $\mathcal{U}$ an open filter of open sets such that $C \subseteq \operatorname{cl}(C \cap U)$ for all $U \in \mathcal{U}$. In sober depos, formal lenses are given by pairs $(C, Q)$ where $C$ is a closed and $Q$ a compact saturated set with nonempty intersection. Such a formal lens is a quasilens if $C \subseteq \operatorname{cl}(C \cap U)$ for all open sets $U$ containing $Q$.

4.3.5. The Heckmann conditions and real lenses. The Heckmann conditions look amazing. But they arise in a natural way from the point of view of real lenses in a dcpo $X$.

Let us fix some notation for this section. Firstly suppose that $X$ is a sober dcpo. Recall that a formal lens is a pair $(C, Q)$ consisting of a closed subset $C$ and a compact saturated subset $Q$ such that $C \cap Q \neq \emptyset$. We denote by $V$ the open set $X \backslash C$ and by $L=C \cap Q$ the 'real' lens associated with the formal lens.

The formal lenses are in a one-to-one correspondence with the $\varphi \in \operatorname{hom}_{0,1}\left(\mathbb{A}^{X}, \mathbb{A}\right)$. If we represent $\varphi$ as a pair $\varphi=\left(\varphi_{1}, \varphi_{2}\right) \in \operatorname{hom}_{0,1}\left(\Sigma_{\vee}^{X}, \Sigma_{\vee}\right) \times \operatorname{hom}_{0,1}\left(\Sigma_{\wedge}^{X}, \Sigma_{\wedge}\right)$ such that $\varphi_{1} \geq \varphi_{2}$ according to Lemma 4.11, then the corresponding formal lens $(C, Q)$ is obtained as $C=X \backslash V$, where $V=\bigcup\left\{U \mid \varphi_{1}(U)=0\right\}$ is the greatest open set with $\varphi_{1}(V)=0$, and $Q=\bigcap\left\{U \mid \varphi_{2}(U)=1\right\}$. Let $L$ be the real lens $L=C \cap Q$. 
A real lens $L$ has many representations by formal lenses. Among them there is a 'least' one, namely $(\mathrm{cl}(L), \uparrow L)$. We would like to characterize those $\varphi \in \operatorname{hom}_{0,1}\left(\mathbb{A}^{X}, \mathbb{A}\right)$ that correspond to these minimal representations. This leads to the Heckmann conditions.

Lemma 4.21. Given a lens $L$ in a dcpo $X$, define maps $\varphi_{1}$ and $\varphi_{2}$ from $\Sigma^{X}$ to $\Sigma$ by

$$
\varphi_{1}(U)=0 \quad \text { iff } \quad L \cap U=\emptyset \quad \text { and } \quad \varphi_{2}(U)=1 \quad \text { iff } L \subseteq U,
$$

then $\varphi_{L}=\left(\varphi_{1}, \varphi_{2}\right)$ is an $\mathbb{A}$-valuation. Moreover $\varphi_{L}$ corresponds to the minimal formal lens $(\mathrm{cl}(L), \uparrow L)$ representing $L$.

Proof. One easily checks that $\varphi_{1}$ and $\varphi_{2}$ are join- and meet-semilattice homomorphisms, respectively, preserving bottom and top. For continuity we have to use the compactness of $L$. Moreover the Heckmann conditions are satisfied. (H1') holds since if $U \cap L=\emptyset$ then $L \subseteq W$ iff $L \subseteq U \cup W$ and (2) holds since if $L \subseteq U$ then $W \cap L=\emptyset$ iff $W \cap U \cap L=\emptyset$. Each of the conditions (H1') and (H2') implies that $\varphi_{1} \geq \varphi_{2}$. Indeed $\varphi_{2}(U)=1$, by (H2'), implies $\varphi_{1}(U)=\varphi_{1}(U \cap X)=\varphi_{1}(X)=1$. Thus, $\varphi_{L}=\left(\varphi_{1}, \varphi_{2}\right)$ is an element of $\operatorname{hom}_{0,1}\left(\mathbb{A}^{X}, \mathbb{A}\right)$.

Since $X \backslash \mathrm{cl}(L)$ is the greatest open set disjoint from $L$, the definition of $\varphi_{1}$ yields $\bigcup\left\{U \mid \varphi_{1}(U)=0\right\}=X \backslash \mathrm{cl}(L)$ and, since the intersection of the open neighborhoods of a set is its saturation, we have $\bigcap\left\{U \mid \varphi_{2}(U)=1\right\}=\uparrow L$.

Of course, one will ask now, whether lenses correspond bijectively to the $\varphi=\left(\varphi_{1}, \varphi_{2}\right) \in$ $\operatorname{hom}_{0,1}\left(\mathbb{A}^{X}, \mathbb{A}\right)$ satisfying the Heckmann conditions. We have seen in 4.3 .2 that this is not so, in general, even for algebraic dcpos. Let us try to find sufficient conditions for this to hold.

Lemma 4.22. Let $X$ be a sober dcpo and suppose that $\varphi=\left(\varphi_{1}, \varphi_{2}\right) \in \operatorname{hom}_{0,1}\left(\mathbb{A}^{X}, \mathbb{A}\right)$ satisfies the Heckmann condition (H1'). For the formal lens $(C, Q)$ associated with $\varphi$ we have $Q=\uparrow(C \cap Q)=\uparrow L$.

Proof. We have to show that $Q=\bigcap\left\{U \mid \varphi_{2}(U)=1\right\}=\uparrow L$. For this, it suffices to show that if $L=Q \cap C \subseteq U$, then $Q \subseteq U$. Since $\varphi_{1}(V)=0$, condition (H1') tells us that $\varphi_{2}(U)=\varphi_{2}(U \cup V)$. Now $Q \cap C \subseteq U$ implies $Q \subseteq U \cup V$. Thus $\varphi_{2}(U \cup V)=1$, whence $\varphi_{2}(U)=1$, which implies $Q \subseteq U$ as desired.

Starting with a $\varphi=\left(\varphi_{1}, \varphi_{2}\right)$ satisfying the Heckmann condition (H2'), we would like to show that $C=\operatorname{cl}(L)$. For this, it suffices to show that if $W \cap L=W \cap C \cap Q=\emptyset$ then $W \cap C=\emptyset$.

If we can find an open set $U$ containing $Q$ such that $W \cap U \subseteq V$, then we are on the safe side. Then indeed $\varphi_{2}(U)=1$. Using (H2') we then have $\varphi_{1}(W \cap U)=\varphi_{1}(W)$. Since $W \cap U \subseteq V$, we have $\varphi_{1}(W \cap U)=0$ and hence, $\varphi_{1}(W)=0$, whence $W \subseteq V$.

But to find an open neighborhood $U$ of $Q$ such that $U \cap W \subseteq V$ is a real problem. We can solve this problem if $X$ is locally compact (for the Scott topology) and coherent. Recall that a topological space is coherent if the intersection of any two compact saturated subsets is compact.

Lemma 4.23. Suppose that $\varphi=\left(\varphi_{1}, \varphi_{2}\right) \in \operatorname{hom}_{0,1}\left(\mathbb{A}^{X}, \mathbb{A}\right)$ satisfies the Heckmann condition (H2'). If $X$ is a locally compact coherent sober dcpo, then $C=\operatorname{cl}(L)$ for the formal lens $(C, Q)$ and the real lens $L=C \cap Q$ associated with $\varphi$.

Proof. Let $x \notin \mathrm{cl}(L)$. We will show: there are an open neighborhood $W$ of $x$ with $W \cap L=\emptyset$ and an open set $U$ containing $Q$ such that $W \cap U \cap C=\emptyset$. 
We then can argue that $\varphi_{1}(W \cap U)=0$. Since $\varphi_{2}(U)=1$, condition (H2') yields $\varphi_{1}(W)=\varphi_{1}(W \cap U)=0$, whence $W \cap C=\emptyset$, which implies $x \notin C$.

Thus let $x \notin \mathrm{cl}(L)$. By local compactness we can find a compact saturated neighborhood $H$ of $x$ disjoint from $\mathrm{cl}(L)$. By local compactness, not only every point but also every compact saturated subset $Q$ has a neighborhood basis of compact saturated neighborhoods $\left(K_{i}\right)_{i}$. The intersections $H \cap K_{i}$ are compact by coherence, and they form a down-directed family such that $\bigcap_{i}\left(H \cap K_{i} \cap C\right) \subseteq H \cap Q \cap C=H \cap L=\emptyset$. Thus, $H \cap K_{i} \cap C=\emptyset$ for some $i$ by [7, Theorem II.1.21(3)]. If $W$ denotes the interior of $H$ and $U$ the interior of $K_{i}$, we have open sets with $W \cap U \cap C=\emptyset$.

Each quasicontinuous dcpo (see [7, Section III-3]) is locally compact and sober according to [7, Proposition III-3.7]. Thus, we can apply the previous results 4.22, 4.23 to coherent quasicontinuous depos.

Proposition 4.24. For coherent quasicontinuous dcpos, in particular for coherent continuous dcpos, there is a one-to-one correspondence between (real) lenses and $\mathbb{A}$-valuations given by $L \mapsto \varphi_{L}$ as in Lemma 4.21.

The preceding proposition covers most of the relevant cases since all FS-domains and, in particular, all retracts of bifinite domains are coherent. According to an unpublished result obtained independently by J. Goubault-Larrecq and A. Jung and by J. D. Lawson and Xi Xiaoyong, the generalizations of FS-domains and retracts of bifinite domains to QFS- and QRB-domains agree with the compact coherent quasicontinuous dcpos, and our previous proposition applies to them, too. The result in the preceding Lemma can be strengthened by means of the following lemma that is well known (see e.g. [7, p. 370] or [1]):

Lemma 4.25. For every lens $L$ in a coherent sober dcpo, $\downarrow L$ is closed.

This Lemma together with the previous one allows one to describe the Plotkin powerdomain over a quasicontinuous coherent dcpo in the classical way as the collection of all (real) lenses $L$ with the Egli-Milner order $L \leq_{E M} L^{\prime}$ iff $L \subseteq \downarrow L^{\prime}$ and $L^{\prime} \subseteq \uparrow L$ (see, e.g. [1] or [7, Theorem IV-8.18]).

There is a second class of dcpos where the Plotkin powerdomain consists of the (real) lenses, the countably based continuous dcpos (see [1, ] or [7, Theorem IV-8.18]). Also in this case the $\mathbb{A}$-valuations are in a one-to-one correspondence with the (real) lenses. It would be desirable to have a proof of the statement in Lemma 4.23 that would cover both the countably based and the coherent case.

\section{Final Remarks}

We conclude with some comments on possible extensions of our results and limitations of our methods.

Plotkin powerdomain via different computational prototypes. We have seen that when choosing the computational prototype $\mathbb{A}$ as $\mathcal{P}(\mathbb{S})$, the Plotkin powerdomain of $\mathbb{S}$, then $\mathbb{A}(X)$ will in general be different from $\mathcal{P}(X)$, the Plotkin powerdomain of $X$. However, what happens if we choose $\mathbb{A}$ as $\mathcal{P}(A)$ for some dcpo $A$ more complex than $\mathbb{S}$, e.g. the domain $\mathbb{T}$ of lifted booleans? Does there exist an $A$ such that for $\mathbb{A}=\mathcal{P}(A)$ the repletion $\mathbb{A}(X)$ always gives rise to $\mathcal{P}(X)$ ? 
Combining probability with nondeterminism. There are other algebraic effects where the methods developed above can be applied. Battenfeld [3] has looked at probabilistic effects. He chooses the extended nonnegative reals $\overline{\mathbb{R}}_{+}$with addition and multiplication by nonnegative scalars $\lambda \in \mathbb{R}_{+}$as computational prototype. Using known results about the extended probabilistic powerdomain $\mathcal{V}(X)$ (C. Jones [14], R. Tix [26]) he shows that $\mathcal{V}(X)$ is the repletion of $X$ for any continuous dcpo $X$. Thus, here again we have the phenomenon that the repletion agrees with the free dcpo-algebra with respect to some natural equational laws for probabilistic choice operators.

There have been extensive investigations on combining probabilistic and nondeterministic effects by Tix, Keimel, Plotkin [27, Mislove [18, Goubault-Larrecq [9]. The free dcpo-algebras have been characterized from an equational point of view. As in Section 4 there is an angelic, a demonic and an erratic case to consider. We conjecture that in the angelic and demonic cases the observationally induced approach leads to the same result as the equational approach, while there is a big gap between the two in the erratic case as in the case of nondeterminism without probability considered in Section 4 .

Limitations. In the example of nondeterministic effects considered in Section 4 we make use of the phenomenon that the semilattice homomorphisms between two semilattices $A$ and $B$ form again a semilattice $\operatorname{hom}(A, B)$, the semilattice operation for homomorphisms being defined pointwise. The repletion of a (continuous) dcpo $X$ was always given by $\operatorname{hom}_{0,1}\left(\underline{R}^{X}, \underline{R}\right)$, where $\underline{R}$ was the computational prototype. An analogous phenomenon occurs when dealing with probabilities and when combining probability with nondeterminism. This is a quite exceptional situation. For example, when dealing with noncommutative monoids, the collection of all homomorphisms between two monoids does not carry any natural monoid structure. Thus, if our computational proptotype is a dcpo with a continuous noncommutative monoid structure, the repletion cannot be $\operatorname{hom}\left(\underline{R}^{X}, \underline{R}\right)$. We will pursue this topic elsewhere.

Predicate transformers. For a dcpo $X$ a predicate is usually meant to be a continuous function $u: X \rightarrow \Sigma$ or, equivalently, an open subset $U$ of $X$. If our computational prototyp is $\underline{R}$, we will consider $\underline{R}$-valued predicates, that is continuous functions $u: X \rightarrow \underline{R}$. Thus, $\underline{R}^{X}$ is the dcpo of all $\underline{R}$-valued predicates on $X$. For dcpos $X$ and $Y$, a predicate transformer will be a continuous map $s: \underline{R}^{Y} \rightarrow \underline{R}^{X}$.

Since in all our considerations the computational monads were kind of 'submonads' of the continuation monad $R^{R^{(-)}}$we consider state transformers to be continuous maps $t: X \rightarrow R^{R^{Y}}$ (transforming an input $x \in X$ to an output $t(x) \in R^{R^{Y}}$.

Exponential transpose establishes a one-to-one correspondence between state transformers and predicate transformers:

$$
\left(R^{R^{Y}}\right)^{X} \cong\left(R^{X}\right)^{R^{Y}}
$$

If $\underline{R}$ is a $\mathcal{D}$ cpo $_{\Omega^{-a l g e b r a}} \operatorname{hom}\left(\underline{R}^{X}, \underline{R}\right)$ is a subdcpo of $\underline{R}^{\underline{R}^{X}}$. The state transformers $t: X \rightarrow \operatorname{hom}\left(\underline{R}^{Y}, \underline{R}\right)$ correspond to the predicate transformers $s \in \operatorname{hom}\left(\underline{R}^{Y}, \underline{R}^{X}\right)$ through exponential transpose:

$$
\operatorname{hom}\left(\underline{R}^{Y}, \underline{R}\right)^{X} \cong \operatorname{hom}\left(\underline{R}^{Y}, \underline{R}^{X}\right)
$$

We apply this to the particular cases of repletion considered in Section 4 . 
Angelic CASE: For all dcpos $X$ and $Y$, the state transformers $t: X \rightarrow \operatorname{hom}_{0,1}\left(\Sigma_{\vee}^{Y}, \Sigma_{\vee}\right)$ are in one-to-one correspondence with those predicate transformers $s: \Sigma_{\vee}^{Y} \rightarrow \Sigma_{\vee}^{X}$ that preserve binary join, bottom and top:

$$
\operatorname{hom}_{0,1}\left(\Sigma_{\vee}^{Y}, \Sigma_{\vee}\right)^{X} \cong \operatorname{hom}_{0,1}\left(\Sigma_{\vee}^{Y}, \Sigma_{\vee}^{X}\right) .
$$

In terms of open sets, these predicate transformers $s$ are characterized by the properties

$$
s(Y)=X, \quad s(\emptyset)=\emptyset, \quad s(U \cup V)=s(U) \cup s(V) .
$$

Demonic CASE: For all continuous depos $X$ and $Y$, the state transformers $t: X \rightarrow$ $\operatorname{hom}_{0,1}\left(\Sigma_{\wedge}^{Y}, \Sigma_{\wedge}\right)$ are in one-to-one correspondence with those predicate transformers $s: \Sigma_{\wedge}^{Y} \rightarrow$ $\Sigma_{\wedge}^{X}$ that preserve binary meets (intersections), bottom and top:

$$
\operatorname{hom}_{0,1}\left(\Sigma_{\wedge}^{Y}, \Sigma_{\wedge}\right)^{X} \cong \operatorname{hom}_{0,1}\left(\Sigma_{\wedge}^{Y}, \Sigma_{\wedge}^{X}\right) .
$$

In terms of open sets, these predicate transformers $s$ are characterized by the properties

$$
s(Y)=X, \quad s(\emptyset)=\emptyset, \quad s(U \cap V)=s(U) \cap s(V) .
$$

ERRATIC CASE: For all compact continuous dcpos $X$ and $Y$, the state transformers $t: X \rightarrow \operatorname{hom}_{0,1}\left(\mathbb{A}^{Y}, \mathbb{A}\right)$ are in one-to-one correspondence with those predicate transformers $s: \mathbb{A}^{Y} \rightarrow \mathbb{A}^{X}$ that preserve $\bullet$, bottom and top:

$$
\operatorname{hom}_{0,1}\left(\mathbb{A}^{Y}, \mathbb{A}\right)^{X} \cong \operatorname{hom}_{0,1}\left(\mathbb{A}^{Y}, \mathbb{A}^{X}\right) \text {. }
$$

While in the angelic and demonic case the predicates are $\Sigma$-valued, this is not so in the erratic case. But we can represent an $\mathbb{A}$-valued predicate $u$ as a pair $u=\left(u_{1}, u_{2}\right)$ of $\Sigma$-valued predicates with $u_{1} \geq u_{2}$. Recall that the members $\varphi \in \operatorname{hom}_{0,1}\left(\mathbb{A}^{Y}, \mathbb{A}\right)$ can be represented as pairs $\varphi=\left(\varphi_{1}, \varphi_{2}\right) \in \operatorname{hom}_{0,1}\left(\Sigma_{\vee}^{Y}, \Sigma_{\vee}\right) \times \operatorname{hom}_{0,1}\left(\Sigma_{\wedge}^{Y}, \Sigma_{\wedge}\right)$ such that $\varphi_{1} \geq \varphi_{2}$. Consequently, the state transformers $t: X \rightarrow \operatorname{hom}_{0,1}\left(\mathbb{A}^{Y}, \mathbb{A}\right)$ can be seen as pairs $t=\left(t_{1}, t_{2}\right)$ of state transformers $t_{1}: X \rightarrow \operatorname{hom}_{0,1}\left(\Sigma_{\vee}^{Y}, \Sigma_{\vee}\right)$ and $t_{2}: X \rightarrow \operatorname{hom}_{0,1}\left(\Sigma_{\wedge}^{Y}, \Sigma_{\wedge}\right)$ such that $t_{1} \geq t_{2}$. Thus, state transformers for the erratic case consist of an angelic and a demonic state transformer, the angelic one dominating the demonic one.

Similarly, using Observation 4.10, every semilattice homomorphism $s: \mathbb{A}^{Y} \rightarrow \mathbb{A}^{X}$ can be seen as a pair $s=\left(s_{1}, s_{2}\right) \in \operatorname{hom}_{0,1}\left(\Sigma_{\vee}^{Y}, \Sigma_{\vee}^{X}\right) \times \operatorname{hom}_{0,1}\left(\Sigma_{\wedge}^{Y}, \Sigma_{\wedge}^{X}\right)$ such that $s_{1} \geq s_{2}$. Thus, predicate transformers for the erratic case consist of an angelic and a demonic predicate transformer, the angelic one dominating the demonic one.

Plotkin powerdomain: The functional representation of the Plotkin powerdomain $\mathcal{P}(X)$ for continuous dcpos $X$ through the Heckmann conditions allows one to characterize the predicate transformers $\mathbb{A}^{Y} \rightarrow \mathbb{A}^{X}$ corresponding to the state transformers $X \rightarrow \mathcal{P}(Y)$ as the continuous maps $s: \mathbb{A}^{Y} \rightarrow \mathbb{A}^{X}$ that satisfy:

$(\mathrm{H} 1)$ if $s(u)(x)=\perp$ then $s(u \vee v)(x)=s(v)(x)$

$(\mathrm{H} 2)$ if $s(u)(x)=\top$ then $s(u \wedge v)(x)=s(v)(x)$.

If one represents $s$ as a pair $\left(s_{1}, s_{2}\right)$ of maps $\Sigma^{X} \rightarrow \Sigma$ these conditions read: $s_{1}$ and $s_{2}$ are continuous maps preserving bottom, top and binary union, resp., intersection, and satisfy:

$\left(\mathrm{H}^{\prime}\right)$ if $s_{1}(u)(x)=0$ then $s_{2}(u \vee v)(x)=s_{2}(v)(x)$

$\left(\mathrm{H} 2^{\prime}\right)$ if $s_{2}(u)(x)=1$ then $s_{1}(u \wedge v)(x)=s_{1}(v)(x)$

Thus, these predicate transformers consist of an angelic component $s_{1}$ and a demonic component $s_{2}$ which are related by the conditions (H1') and (H2'). 


\section{REFERENCES}

[1] Samson Abramsky and Achim Jung. Domain theory. volume 3 of Handbook of Logic in Computer Science, pages 1-168. Clarendon Press, Oxford, 1994.

[2] Ingo Battenfeld. Observationally-induced effects in cartesian closed categories. Electr. Notes Theor. Comput. Sci., 286:43-56, 2012.

[3] Ingo Battenfeld. Observationally-induced Algebras in Domain Theory. Electr. Notes Theor. Comput. Sci., 301: 21-37, 2014.

[4] Ingo Battenfeld and Matthias Schröder. Observationally-induced effect monads: Upper and lower powerspace constructions. Electr. Notes Theor. Comput. Sci., 276:105-119, 2011.

[5] Ingo Battenfeld and Matthias Schröder. Observationally induced upper and lower powerspace constructions. 2012. submitted for publication.

[6] Garrett Birkhoff. Lattice Theory, American Mathematical Society Colloquium Publications vol. XXV, 3rd edition, (1967).

[7] Gerhard Gierz, Karl Heinrich Hofmann, Klaus Keimel, Jimmie Lawson, Michael Mislove, and Dana S. Scott. Continuous Lattices and Domains. Cambridge University Press, Cambridge, 2003.

[8] Jean Goubault-Larrecq. Prevision domains and convex powercones. In Roberto M. Amadio, editor, FoSSaCS, volume 4962 of Lecture Notes in Computer Science, pages 318-333. Springer, 2008.

[9] Jean Goubault-Larrecq. De Groot duality and models of choice: angels, demons and nature. Mathematical Structures in Computer Science, 20(2):169-237, 2010.

[10] Reinhold Heckmann. Abstract valuations: A novel representation of Plotkin power domain and Vietoris hyperspace. Electr. Notes Theor. Comput. Sci., 6:160-173, 1997.

[11] Martin Hyland First Steps in Synthetic Domain Theory Proc. of Category Theory 1990, SLNM 1488, pp. 131-156, 1991.

[12] Martin Hyland, Paul Blain Levy, Gordon D. Plotkin, and John Power. Combining algebraic effects with continuations. Theor. Comput. Sci., 375(1-3):20-40, 2007.

[13] Martin Hyland, Gordon D. Plotkin, and John Power. Combining computational effects: commutativity \& sum. In Ricardo A. Baeza-Yates, Ugo Montanari, and Nicola Santoro, editors, IFIP TCS, volume 223 of IFIP Conference Proceedings, pages 474-484. Kluwer, 2002.

[14] Claire Jones. Probabilistic Non-determinism. PhD thesis, LFCS, University of Edinburgh, 1989.

[15] Simon Peyton Jones, editor. Haskell 98 language and libraries. Cambridge University Press, Cambridge, 2003. The revised report, J. Funct. Programming 13 (2003), no. 1.

[16] Klaus Keimel. Topological cones: Foundations for a domain theoretical semantics combining probability and nondeterminism. Electr. Notes Theor. Comput. Sci., 155:423-443, 2006.

[17] Klaus Keimel. On the equivalence of state transformer semantics and predicate transformer semantics. Proceedings of the Workshop Informatics and Information Technologies in Education: Theory, Practice, Didactics, Novosibirsk, 1:78-104, 2012.

[18] Michael Mislove. Nondeterminism and probabilistic choice: Obeying the law. In: Proc. 11th Conf. Concurrency Theory (CONCUR'00), LNCS 1877: 350-364. Springer Verlag, 2000.

[19] Eugenio Moggi. Computational lambda-calculus and monads. In Proceedings, Fourth Annual Symposium on Logic in Computer Science, 5-8 June, 1989, Asilomar Conference Center, Pacific Grove, California, USA, pages 14-23. IEEE Computer Society, 1989.

[20] Gordon D. Plotkin and John Power. Notions of computation determine monads. In Mogens Nielsen and Uffe Engberg, editors, FoSSaCS, volume 2303 of Lecture Notes in Computer Science, pages 342-356. Springer, 2002. Erratum available at: http://homepages.inf.ed.ac.uk/gdp/publications/.

[21] Gordon D. Plotkin and John Power. Computational effects and operations: An overview. Electr. Notes Theor. Comput. Sci., 73:149-163, 2004.

[22] Matthias Schröder and Alex Simpson. Probabilistic observations and valuations: (extended abstract). Electr. Notes Theor. Comput. Sci., 155:605-615, 2006.

[23] Alex Simpson. Probabilistic observations and valuations. Talk given at: MFPS XXI, University of Birmingham, 2005. Slides: http://homepages.inf.ed.ac.uk/als/.

[24] Alex Simpson. Beyond classical domain theory. Talk given at: MFPS XXIII, University of Birmingham, 2007. Slides: http://homepages.inf.ed.ac.uk/als/.

[25] Michael B. Smyth. Topology. volume 1 of Handbook of Logic in Computer Science, pages 641-761. Clarendon Press, Oxford, 1992. 
[26] Regina Tix. Stetige Bewertungen auf topologischen Räumen (Continuous valuations on topological spaces, in German). Master's thesis, TU Darmstadt, 1995.

[27] Regina Tix, Klaus Keimel, and Gordon D. Plotkin. Semantic domains for combining probability and non-determinism. Electr. Notes Theor. Comput. Sci., 129:1-104, 2005.

This work is licensed under the Creative Commons Attribution-NoDerivs License. To view a copy of this license, visit http://creativecommons.org/licenses/by-nd/2.0/ or send a letter to Creative Commons, 171 Second St, Suite 300, San Francisco, CA 94105, USA, or Eisenacher Strasse 2, 10777 Berlin, Germany 